\title{
Opposing selective forces operating on human- specific duplicated TCAF genes in Neanderthals and humans
}

\section{PingHsun Hsieh}

University of Washington School of Medicine

\section{Vy Dang}

University of Washington School of Medicine

\section{Mitchell Vollger}

University of Washington School of Medicine

\section{Yafei Mao}

University of Washington

\section{Tzu-Hsueh Huang}

University of Washington School of Medicine

\section{Philip Dishuck}

Univ of WA https://orcid.org/0000-0003-2223-9787

\section{Carl Baker}

University of Washington School of Medicine

\section{Stuart Cantsilieris}

University of Washington School of Medicine

\section{Alexandra Lewis}

University of Washington School of Medicine

\section{Katherine Munson}

University of Washington https://orcid.org/0000-0001-8413-6498

\section{Melanie Scofield}

University of British Columbia

\section{AnneMarie Welch}

University of Washington School of Medicine

\section{Jason Underwood}

Pacific Biosciences (United States)

Evan Eichler ( $\nabla$ eee@gs.washington.edu )

University of Washington School of Medicine https://orcid.org/0000-0002-8246-4014 
Keywords: Human-specific genes, thermal regulatory gene duplications, natural selection, archaic and modern humans

Posted Date: October 1st, 2020

DOI: https://doi.org/10.21203/rs.3.rs-77798/v1

License: (c) (1) This work is licensed under a Creative Commons Attribution 4.0 International License. Read Full License

Version of Record: A version of this preprint was published at Nature Communications on August 25th, 2021. See the published version at https://doi.org/10.1038/s41467-021-25435-4. 
1 Opposing selective forces operating on human-specific duplicated TCAF genes in Neanderthals and

2 humans

3 PingHsun Hsieh, ${ }^{1 *}$ Vy Dang,,${ }^{1,}$ Mitchell R. Vollger, ${ }^{1}$ Yafei Mao, ${ }^{1}$ Tzu-Hsueh Huang, ${ }^{1}$ Philip C. Dishuck, ${ }^{1}$

4 Carl Baker ${ }^{1}$, Stuart Cantsilieris, ${ }^{1,+}$ Alexandra P. Lewis, ${ }^{1}$ Katherine M. Munson, ${ }^{1}$ Melanie Sorensen, ${ }^{1}$

5 AnneMarie E. Welch, ${ }^{1, \ddagger}$ Jason G. Underwood, ${ }^{1,2}$ Evan E. Eichler ${ }^{1,3^{*}}$

6

7

$8 *$ Co-corresponding authors

9

10 Correspondence: hsiehph@uw.edu

11

eee@gs.washington.edu

12 Affiliations:

13 1. Department of Genome Sciences, University of Washington School of Medicine, Seattle, WA, USA

14 2. Pacific Biosciences (PacBio) of California, Incorporated, Menlo Park, CA, USA

15 3. Howard Hughes Medical Institute, University of Washington, Seattle, WA, USA

$16 \uparrow$ Current address: Institute for Cell and Molecular Biology, University of Texas, Austin, TX, USA

17 \$ Current address: Centre for Eye Research Australia, Department of Surgery (Ophthalmology),

18 University of Melbourne, Royal Victorian Eye and Ear Hospital, East Melbourne, VIC, Australia

$19 \neq$ Current address: Brain and Mitochondrial Research, Murdoch Children’s Research Institute, Royal

20 Children's Hospital, Melbourne, VIC, Australia

21

22 Keywords: Human-specific genes, thermal regulatory gene duplications, natural selection, archaic and 23 modern humans 


\section{SUMMARY}

26 TRP channel-associated factor 1/2 (TCAF1/TCAF2) proteins antagonistically regulate the cold-sensor

27 protein TRPM8 in multiple human tissues. Understanding their significance has been complicated given

28 the locus spans a gap-ridden region with complex segmental duplications in GRCh38. Using long-read

29 sequencing, we sequence-resolve the locus, annotate full-length TCAF models in human and nonhuman

30 primate genomes, and show substantial human-specific TCAF copy number variation. We identify two

31 human super haplogroups, $\mathrm{H} 4$ and H5, and establish that TCAF duplications originated $\sim 1.7$ million years

32 ago but diversified only in Homo sapiens by recurrent structural mutations that altered TCAF copy

33 number and regulation. Conversely, in all archaic-hominin samples the fixation for a specific $\mathrm{H} 4$

34 haplotype without duplication is likely due to positive selection. The significant, positive effect of H4 on

35 TCAF2 expression in modern-day humans with candidate associations for hypothyroidism, nerve

36 compression, and diabetes suggests TCAF diversification among hominins potentially in response to cold

37 or dietary adaptations. 


\section{INTRODUCTION}

40 Gene duplication contributes significantly to molecular evolution by providing the raw material for

41 genetic novelty and organismal adaptation ${ }^{1,2}$. In the human lineage, recently duplicated regions

42 corresponding to segmental duplications (SDs) are known to give rise to new genes contributing to

43 synaptogenesis, neuronal migration, and neocortical expansion ${ }^{3-7}$ that distinguish human from other ape

44 species. Although relatively few in number, some of the largest genetic changes that differentiate humans

45 from archaic hominins involve gene-rich SDs ${ }^{8,9}$. Among human-specific SD genes, the duplications of

46 TCAF 1/TCAF2 are particularly intriguing. These genes encode TRP channel-associated factors that bind

47 to the protein TRPM8 (transient receptor potential cation channel subfamily M member 8) — the ion

48 channel acting as thermal sensor in peripheral somatosensory neurons ${ }^{10}$ and are thought to be under

49 positive selection in Eurasian populations ${ }^{11}$. Both TCAF1 and TCAF2 proteins interact directly with the

50 TRPM8 channel but have antagonistic effects in TRPM8 gating and trafficking into the plasma

51 membrane: while TCAF1 facilitates the TRPM8 channel opening and migration, the activity of TRPM8 is

52 completely suppressed by TCAF2 ${ }^{12}$. These results suggest that the relative abundance of TCAF proteins

53 and their competition in binding TRPM8 is likely critical in physiological regulation of the channel

54 activity ${ }^{12}$.

55 The TCAF family originated from an ancient gene duplication event at the basal of mammalian

56 phylogeny and stayed as single-copy genes throughout much of their evolution ${ }^{8,12}$. Within the human

57 lineage, subsequent duplications over the last few million years have changed the copy number of both

$58 T C A F 1$ and TCAF2. In the human reference genome GRCh38, TCAF1 and TCAF2 are embedded and

59 span within a complex region of large, highly identical SDs (>99.5\%) consisting of $>250$ thousand base

60 pairs (kbp) sequence and an annotated gap at chromosome 7q35. Studying these SDs is particularly

61 problematic because of the high sequence identity and missing sequences in reference genomes ${ }^{13-15}$.

62 Using large-insert bacterial artificial chromosome (BAC) clones from a haploid hydatidiform mole cell

63 line, we recently assembled a sequence-resolved haplotype spanning over this region and characterized

64 three sets of SDs present in both direct and indirect orientations. Copy number estimations from read- 
65 depth analyses suggest that TCAF duplications are completely missing in archaic hominins, such as

66 Neanderthal and Denisovan, and nonhuman great apes ${ }^{8}$. In addition, while their sequence analysis

67 suggested that a single full-length $T C A F 1$ and $T C A F 2$ exist at the locus, respectively, additional

68 TCAF1/TCAF2 copies appear to be truncated or incomplete ${ }^{8}$.

69 In the present study, we systematically explore the haplotype structure of the TCAF locus in order 70 to study its diversity, annotate the genes, and infer its evolutionary history in the context of selection. We

71 generate 15 sequence-resolved haplotypes from both human and nonhuman primate BAC libraries as well

72 as full-length non-chimeric (FLNC) transcript data from seven tissues using the Pacific Biosciences

73 (PacBio) single-molecule, real-time (SMRT) long-read sequencing technology. We leverage a large

74 collection of over 1,100 publicly available high-coverage Illumina genomes from modern, and archaic

75 humans as well as nonhuman primate samples and document the global diversity of TCAF SDs. Finally,

76 we integrate both phylogenetic and population genetic inference approaches to reconstruct the evolution

77 of the TCAF SDs in primates and identify putative signals of balancing selection in Native American,

78 Melanesian, and Siberian populations as well as positive selection in both Neanderthal and Denisovan.

79 We provide evidence for differential expressed quantitative trait loci (eQTLs) between two TCAF super

80 haplogroups in thyroid, whole blood, tibial nerve, spleen, and lung tissues. In addition, our association

81 analysis using the UK Biobank data suggest that the TCAF super haplogroups are likely associated with

82 hypothyroidism, diabetes, and nerve-related traits in some human populations. The study provides one of

83 the most complete genetic investigations of human-specific SDs shedding potential new insights into

84 structural adaptations important in thermal regulation.

85

\section{RESULTS}

87 Diversity of TCAF copy number in human and nonhuman primates. We applied a read-depth-based

88 genotyper ${ }^{16}$ to reevaluate copy number diversity of TCAF SDs (duplication segments A, B, and C;

89 Figure 1A) in a collection of recently published diverse human and nonhuman samples ${ }^{17-23}$. The set

90 includes high-coverage Illumina genomes from a global panel of 1,102 human samples, four archaic 
91 hominins, and 71 nonhuman great apes. Consistent with previous studies ${ }^{8}$, all archaic hominin and

92 nonhuman primate samples are fixed for diploid copy number $(\mathrm{CN}) 2$ (Figure 1B). Among contemporary

93 modern human samples, the overall diploid copy number estimates of the three TCAF SDs range from

94 two to eight copies and are highly correlated among each other (Figure S1), indicating that these three

95 SDs likely appear as a cassette in the evolution of this locus. In contrast to archaic humans where there is

96 a prediction of a single copy of this locus, we estimate that $~ 98 \%$ of modern humans carry more than two

97 copies (Figures 1B, S1), suggesting that most of the diversity emerged during the divergence of

98 hominins.

99 Among humans, we observed a wide range of variation across different geographic locations in

100 contemporary human populations (Figure 1B-C). While the median copy numbers are consistently CN4

101 (with the exception of Native Americans [median = 3]), African samples on average carry more copies

102 (mean $\mathrm{CN}=4.6$ ) than other populations (Figure 1B). We tested for copy number stratification by

103 applying both the $V_{S T}$ statistic ${ }^{24}$ and $\mathrm{CN}$ differentiation test ${ }^{25}$. While there is little evidence for population

104 differentiation among pairs of super populations, a reduction in diploid CN among Native American

105 samples distinguishes this group from all others $\left(V_{S T}>0.21\right.$, Bonferroni $p$ value for the CN differentiation

106 test $<0.0014$; Table S1).

107 The observation that all diploid CN2 samples (i.e., without duplications) are present solely among

108 non-African populations raises the question as to whether it is due to archaic introgression. To address

109 this, we compared single-nucleotide variation at $20 \mathrm{kbp}$ unique diploid sequences flanking the TCAF SD

110 locus (chr7:143,501,000-143,521,000, chr7:143,875,000-143,895,000 [GRCh38]) between archaic and

111 modern human genomes. In general, the archaic flanking haplotypes are similar to those present in

112 modern human samples, including the AFR samples (Figure S2). Focusing on sites that are fixed in

113 derived alleles in either the Neanderthal or Denisovan samples, the numbers of derived allele counts in

114 the CN2 samples are comparable to those drawn randomly from African samples $(\mathrm{p}=0.276 ; 1,000$ non-

115 parametric bootstrap samples). These results suggest that non-African CN2 haplotypes unlikely arose as a

116 result of introgression from Neanderthal or Denisovan. 
118 Characterization of TCAF haplotype diversity using long-read BAC-based assemblies. These near-

119 perfect duplications have hampered not only genome assembly but also the characterization of underlying

120 genetic diversity as well as our understanding of the TCAF evolution. An investigation of four recently

121 published long-read whole-genome assemblies, for example, showed that all four failed to create

122 contiguous sequences for both haplotypes spanning over the TCAF SDs (Figure S3, Table S2). To

123 resolve this, we selected large-insert BAC clones and used PacBio long-read sequencing to generate 15

124 high-quality sequence-resolved haplotypes from eight human samples and three nonhuman primates,

125 including chimpanzee, gorilla, as well as an Old World rhesus macaque monkey (Table 1, Methods).

126 A sequence comparison analysis among these newly generated haplotypes confirms the complex

127 organization of these TCAF $\mathrm{SDs}^{8}$ and further reveals considerable structural diversity in humans when

128 compared to nonhuman primates (Figure 2 and S4-S12). While all the nonhuman primate haplotypes

129 carry a single copy for the TCAF SD cassette and single full-length TCAF1 and TCAF2 genes (Figures

130 S4, S11-S12), among the 12 human haplotypes, we identify five distinct haplogroups that carry one to

131 three copies for the SD cassettes, which range from 145-406 kbp in length (Figure 2, Table 1). The two

132 largest human haplogroups (Haplogroups 4 and 5) both carry three copies of the SD cassette (Figures 3,

133 S5) but differ by a $100 \mathrm{kbp}$ inversion (Haplogroup5:337,305-437,635; Figure S5). The Haplogroup 5

134 organization is mostly consistent with the current human reference genome (GRCh38), which has a gap in

135 the middle of expanded complex SDs in the reference (Figure 3). The newly generated Haplogroup 5

136 sequence, thus, eliminates the gap by adding 103,616 bp (Haplogroup5:216,541-320,157) in the human

137 reference.

138 Most of the sequence-resolved haplotypes (8 out of 15) consist of two copies of the TCAF SD

139 cassette (Haplogroups 2 and 3; Figure 2, S6-S9). These two haplogroups can be structurally distinguished

140 by a similar $100 \mathrm{kbp}$ inversion as identified between Haplogroups 4 and 5. We, therefore, use this

141 inversion polymorphism to classify two super haplogroups (H4 and H5, Figure 2). The shortest human

142 BAC haplotypes (Haplogroup 1; Figures 2 and S10) that we assembled consist of a single copy of the 
143 SD cassette and are similar in structure to those found in nonhuman primates. To further refine the

144 relationship among these assembled haplotypes, we performed a series of sequence alignment analyses

145 among different pairs of haplotypes (Figure 2). Based on the organization of the TCAF SDs, we

146 conservatively determined that Haplogroups 2 and 5 are closely related and so are Haplogroups 3 and 4.

147 Among Haplogroup 2, two subgroups, Haplogroup 2-1 $(\mathrm{n}=1)$ and Haplogroup 2-2 $(\mathrm{n}=5)$, were further

148 separated and likely emerged due to two independent events, where each involves a different $130 \mathrm{kbp}$

149 deletion, with respect to Haplogroup 5 (Figure 2, Table S3). Similarly, Haplogroup 3 can also be further

150 classified into two subgroups, Haplogroup 3-1 and Haplogroup 3-2, because we predict that they likely

151 derived from two different large deletions with respect to Haplogroup 4 (Figure 2, Table S3). Finally,

152 while Haplogroup1 has a similar structure to the nonhuman primate haplotypes, we showed that this

153 group is more closely related to Haplogroup 3 and likely a result of a deletion event over Haplogroup 3

154 (Figure S10, Table S3).

155 The breakpoints of these six large structural differences map within nearly identical (>99.5\%)

156 SDs, consistent with the action of non-allelic homologous recombination (NAHR) (Table S3). By

157 identifying the longest, high-identity sequences around the putative breakpoints (Table S3), we show that

158 four associate with DupA SDs, while two map around DupC SDs. For example, the inferred inversion

159 breakpoints of Haplogroup 4 are immediately flanking two inversely oriented, nearly identical sequences

160 within DupC SDs ( 39.6 kbp; sequence identity > 99.8\%). In addition, our analysis of sequence

161 composition reveals the presence of nearly identical LINE/SINE elements in sequences flanking the

162 putative breakpoints (Table S3). The inferred breakpoints for the deletion event between Haplogroups 3-1

163 and 4 overlap with two perfectly identical (100\%) $2.5 \mathrm{kbp}$ CTAGE (Cutaneous T Cell Lymphoma-

164 Associated Antigen 1) family sequences (Figure S8, Table S3). Remarkably, most of the deletion events

165 are similar in size ranging in length from 129.7 to $134.4 \mathrm{kbp}$. Thus, our data highlight a rapid

166 evolutionary process that involves large-scale duplication, deletion, and inversion events via NAHR but

167 where deletion events are constrained. 
Discovery of new TCAF genes/isoforms. The newly generated long-read BAC haplotypes reported allow us to revisit $T C A F$ annotation. Functional studies have shown that $T C A F 1 / T C A F 2$ are highly expressed in

171 the prostate, brain, esophagus, prostate, and skin tissues ${ }^{12}$ (GTEx Portal, https://gtexportal.org). To this

172 end, we targeted capture of FLNC TCAF transcripts and generated 480,700 FLNC transcripts from six

173 human tissues, including dorsal root ganglion, esophagus, fibroblast, skin, fetal brain, and testis, as well

174 as 50,885 FLNC transcripts from a chimpanzee lymphoblast cell line (Table S4). TCAF transcript on-

175 target rates range from the lowest in the testis sample (2.2\%) to the highest for the chimpanzee

176 lymphoblast sample (15.5\%). To identify TCAF models, we aligned high-quality FLNC transcripts to the

177 assembled haplogroup sequences and only retained transcripts that have complete open reading frames

178 with >100 amino acids (aa) in length (Methods). Of note, we followed the previously described

179 nomenclature ${ }^{8}$ to delineate TCAF models and isoforms. Overall, our analysis indicates the expression of

$180 T C A F$ genes in a wide variety of tissues and reveals new TCAF paralogs as well as a variety of new

181 isoforms (Figures 3, S5-S13; Table S5). In particular, we recovered only single isoforms for individual

182 genes in the TCAF1 subfamily. In contrast, the TCAF2 subfamily shows much more diversity with 10, 2,

183 and 4 isoforms being identified for $T C A F 2 A, T C A F 2 C 1$, and $T C A F 2 C 2$, respectively. With respect to the

184 current annotation in the human reference genome (GRCh38), all haplotypes confirm that TCAF2A (or

185 TCAF2 in RefSeq Release 200) is incorrectly annotated due to the presence of a gap at this locus (Figure

186 3). The gene models and isoforms reported here represent the most correct and comprehensive

187 annotations for the TCAF families (Table S5).

188 Compared to the chimpanzee, every additional copy of an SD cassette identified among human

189 haplotypes is associated with an additional copy of TCAF1A and TCAF2C paralogs, although they are

190 incomplete copies to $T C A F 1 B$ and $T C A F 2 A$, respectively (Figures 3, S4-S12). Specifically, all

191 TCAF1A1/TCAF1A2 paralogs match to the last seven exons of TCAF1B, while the TCAF2C1/TCAF2C2

192 paralogs consist of the first three exons of TCAF2A (Figure 3). In addition, the predicted breakpoints

193 between Haplogroup 2-2 and Haplogroup 5 coincide with the TCAF1A1 and TCAF1A2 paralogs. The

194 sequence alignment analysis shows that the breakpoint at Haplogroup 2-2 spans from the third to the fifth 
introns, and thus, the TCAF1A1 copy of Haplogroup 2-2 is a fusion version of the TCAF1A1 and TCAF1A2 copies of Haplogroup 5 (Figure S8). Because the amino acid sequences encoded from these

197 three paralogs are $100 \%$ identical, this suggests the actual breakpoints are within one of the three introns.

198 Finally, the $100 \mathrm{kbp}$ inversion between Haplogroup 4 and Haplogroup 5 raises the question whether the

199 large-scale structural variation event has any effect on TCAF coding sequences. Notably, the inferred

200 inversion breakpoints map to the second introns of TCAF2A and TCAF2C2. There are only two

201 differences in the TCAF2A exon alignment between Haplogroups 4 and 5: one is a synonymous change in

202 the second exon, and the other causes a nonsynonymous change (R479Q) in the third exon, which is

203 beyond the inferred breakpoints (Figure S13). Thus, our results predict that this inversion shuffles the last

204 six exons of $T C A F 2 A$, which were ligated with the first two exons of different $T C A F 2 A / T C A F 2 C 2$

205 paralogs on the two haplotypes without disrupting or altering the coding sequence potential of

TCAF2A/TCAF2C2.

It should be noted that although the structural changes largely increase and decrease dosage of individual TCAF family members with relatively few predicted amino-acid differences, there is evidence

210 for candidate sites of interlocus gene conversion (IGC) among paralogs (Figure S14) and observed an

211 IGC site that overlaps with a strong H3K27Ac signal (left white box, Figure 1A) an epigenetic signature

212 for enhancers. Using paralog-specific copy number genotypes (Methods), we found a reduction in copy

213 number at this locus (i.e., the acceptor site; chr7:143,615,002-143,624,482, GRCh38; the left white box,

214 Figures 1A and S15), which, interestingly, is significantly negatively correlated with an increase in copy

215 number at its paralogous locus (the donor site; chr7:143,833,163-143,842,658; the right white box,

216 Figures 1A and S15) (Pearson's correlation $=-0.3$, $\mathrm{p}$ value $=5 \times 10^{-16}$ ) in multiple continental populations

217 (Figure S16). This result is consistent with the hypothesis of an IGC event copying the donor sequence

218 over the acceptor locus, resulting in reciprocal copy number changes at these two loci. Interestingly, we

219 find that this paralog-specific copy number at the donor locus is negatively correlated $(\mathrm{R}=-0.18, \mathrm{p}$

220 value $=3.6 \times 10^{-7}$ ) with the (absolute) latitudinal location of human populations while the relationship for 
221 the acceptor site is positive $\left(\mathrm{R}=0.23\right.$, $\mathrm{p}$ value $=2.5 \times 10^{-11}$; Figure $\left.\mathbf{S 1 7}\right)$. While the observed correlations

222 between latitudes of individual populations and paralog-specific copy numbers at the IGC sites may be

223 the result of natural selection, such strong correlations are relatively common among similar loci across

224 the genome ( $\mathrm{p}$ value $=0.22 ; 1,000$ random SD loci of genomic shuffling), suggesting demographic

225 history among populations also likely contributing to this observation.

227 Evolution of TCAF segmental duplications. To reconstruct evolutionary origin of the TCAF haplotypes

228 in modern humans, we leveraged these high-quality human and nonhuman primate haplotypes for

229 phylogenetic reconstruction (Tables 1 and S6). The mean sequence identities for DupA, DupB, and

230 DupC SDs are high among human haplogroups (99.80\% [s.d.: 0.04], 99.71\% [s.d.: 0.10], and 99.85\%

231 [s.d.: 0.05], respectively) (Figure S18), while the sequence divergence between human and nonhuman

232 primate TCAF haplotypes are compatible to published whole-genome estimates (Figure S18) ${ }^{26,27}$.

233 Together, these findings are consistent with a recent TCAF SD expansion or IGC. We considered each of

234 the duplicated segments independently guarding against confounders such as IGC and non-allelic

235 homologous recombination events. Because several SDs represent hybrids of two SD paralogs due to

236 NAHR fusion events (Figure 2), for example, we restricted our analysis to the larger segments of hybrid

237 SDs. To minimize the effects of IGC (Figure S14) ${ }^{8}$, we applied GENECONV (v1.81a) to exclude sites

238 corresponding to $80.5 \%, 61.7 \%$, and $70.6 \%$ of DupA, DupB, and DupC sequences, respectively (Table

239 S7). Phylogenetic reconstruction and dating estimates on this filtered set showed that all human copies

240 form a single clade with a $100 \%$ posterior support and coalesce at 1.06 (95\% C.I.:0.87-1.26), 1.72 (95\%

241 C.I.: 1.26-2.21), and 1.47 (95\% C.I.: 0.74-2.54) million years ago (Mya), for DupA, DupB, and DupC

242 SDs, respectively (Figures 4A and S19-S21). While a few differences in topology were noted among the

243 inferred phylogenies (Figures S19-S21), these estimates, with the exception of DupB, overlapped

244 coalescent estimates obtained from a $12.3 \mathrm{kbp}$ single-copy unique region (Figure 2, the right panel),

245 which suggests that the two supergroups: H5 (Haplogroup 5, Haplogroup 2-1, and Haplogroup 2-2) and

246 H4 (Haplogroup 4, Haplogroup 3-1, Haplogroup 3-2, and Haplogroup 1) diverged from each other 0.73 
247 Mya (kya; 95\% C.I.: 0.46-1.03) (Figure S22A). Importantly, these findings suggest that the duplications

248 began to occur before humans and archaic hominins diverged.

To further refine our inferences on the evolution of $T C A F$, we realigned genome sequence data

250 from four archaic hominins along with human and chimpanzee samples to a custom, gapless human

251 reference chromosome 7 using GRCh38 and sequence-resolved BAC haplotypes to generate a joint

252 single-nucleotide variant call set (Methods). Due to the limitations of short-read data, we restricted this

253 analysis to three unique regions (52.3 kbp in total) flanking and internal to the TCAF locus (Figures 1

254 and 2). We identified 1,275 single-nucleotide variants (SNVs) (QV>20) and used these to

255 computationally phase and to construct haplotypes from 802 samples (Figures S2 and S23). Using these

256 archaic hominin haplotypes with the sequences of the seven BAC haplogroups, we estimate the time to

257 the most recent common ancestor (TMRCA) of all hominins being 0.78 Mya (95\% C.I.:0.55-1.03 Mya),

258 which is consistent with that reported above. Interestingly, all archaic haplotypes and the supergroup H4

259 are more closely related than either is to the supergroup H5, and the clade of archaic and supergroup H4

260 haplotypes coalesces $\sim 0.53$ Mya (Figure 4B). Thus, our results show that as early as 1.72 Mya the TCAF

261 locus (DupB SD) began to duplicate and that by 0.73 Mya most of the of the TCAF haplotype diversity

262 that we observe in modern-day humans had already emerged. Archaic hominins, in contrast, show a

263 reduction in genetic diversity without the associated copy number duplication diversity found in modern

264 humans (Figure 4C).

265

266 Hominin diversity and natural selection of TCAF haplotypes. We used the combined haplotypes

267 constructed from the $53.2 \mathrm{kbp}$ of unique regions to further explore genetic diversity among archaic and

268 present-day hominins. A haplotype-based principal component analysis (PCA) reveals distinct clusters

269 (Figures S24-S25) corresponding in part to the two human haplogroups H4 and H5 identified from the

270 BAC sequence analysis (Figure S25). Using a supervised clustering method based on haplotype PCA and

271 machine-learning algorithm t-SNE ${ }^{28}$ for dimensionality reduction and visualization (Methods), we show

272 that contemporary hominin haplotypes can be classified into 12 different clusters (Figures 5A and S26) 
273 with an estimated haplotype heterozygosity of 0.885 (Methods), which is much higher than the reported

274 genome-wide estimated mean of $0.534{ }^{29}$. Phylogenetic reconstruction using 10 haplotypes drawn at

275 random from each cluster reproducibly reconstructs a maximum likelihood tree closely resembling this

276 cluster-based relationship (Figure 5B). Considering the two flanking and unique regions independently

277 also confirms a phylogeny of 12 distinct clusters with different BAC haplogroup representing different

278 clusters (Figures S26-S27). Among humans, no single population is restricted to a specific cluster,

279 although some clusters appear enriched. For example, clusters 2 and 7 largely consist of African (60.3\%)

280 and South Asian (50.6\%) haplotypes, respectively (Figure S28). Assuming an equal split of the overall

281 diploid $\mathrm{CN}$ estimates between the two haplotypes in an individual, we found significant differences in

$282 T C A F$ copy number among haplotype clusters (Bonferroni corrected p values < 0.05; Mann-Whitney $U$

283 tests) (Figure S29).

In sharp contrast to the extensive haplotype diversity in modern human samples, the eight archaic hominin haplotypes are virtually identical belonging to a single homogenous group (Figures 5, S24-S29). Under a panmictic model and assuming an ancestral hominin origin of $\sim 0.73$ Mya for TCAF haplotypes, it

287 is highly unlikely that all eight archaic haplotypes would form a single cluster given modern-day human 288 diversity ( $\mathrm{p}$ value $=2.1 \times 10^{-7}, 100 \times 10^{6}$ permutations). To formally test whether natural selection plays a 289 role in these unusual patterns in both modern and archaic humans, we performed a selection test using the 290 Tajima's $D$ statistic and coalescent simulations under plausible demographic models to assess

291 significance (Methods). The results suggest significant distinct signals of selection in humans and archaic 292 hominins separately (Figure 5C). Among modern human populations, Native American, Melanesian, and 293 Siberian populations show evidence for excess of heterozygosity and significantly larger Tajima's $D$ s 294 compared to those from coalescent simulations under neutral demographic models (Figure 5C); though 295 the distributions for Melanesians and Siberians center on zero. Especially among Native Americans 296 (Tajima's $D=1.24$, p value $=4.3 \times 10^{-9}$ ), these results are consistent with the action of balancing selection 297 at this locus (Figure 5C). In contrast, the Tajima's $D$ distribution of the archaic hominin samples are 298 strictly negative and significantly lower than the neutral expectation, and this result holds even when the 
Denisovan sample was removed (Tajima's $D=-1.05$; p value $\left.<1 \times 10^{-6}\right)($ Figure 5C). Our results suggest that the observed archaic hominin haplotype variation pattern is unlikely explained by their demographic 301 history, thus, favoring a hypothesis of positive selection.

These dramatic differences in diversity and potentially distinct selection forces pose the question 303 of whether there are any functional differences among the different TCAF haplogroups. Because our 304 structural analyses suggested dosage and regulatory differences among common haplogroups (Figures 305 S28-S29), we investigated TCAF2 expression using the GTEx multi-tissue data (release v8) and possible 306 genetic associations between traits and TCAF haplotypes using the UK Biobank data. Based on the BAC 307 references, we identified four tagging SNVs in the unique diploid region embedded among the TCAF SDs 308 that could distinguish super haplogroups H4 and H5 (Figure 6). Because the two super haplogroups 309 structurally differ by the $100 \mathrm{kbp}$ inversion, we expect a reduction of homologous recombination events 310 between the two groups and therefore an increase in linkage disequilibrium (LD) in hominins. As 311 expected, we observe substantial LD across all three unique diploid regions in all archaic and modern 312 samples and confirm nearly complete LD among the four tagging SNVs $\left(D^{\prime}>0.98, R^{2}>0.89\right)$ (Figure 313 S30). Using these data, we again found that all archaic samples are fixed for the H4 supergroup and 314 inferred the frequencies of $\mathrm{H} 4, \mathrm{H} 5$, and other forms in modern humans being $57.1 \%, 40.3 \%$, and $2.6 \%$, 315 respectively. While the H4 haplogroup is found at high frequency among non-Africans, especially in 316 Melanesians (72\%), some Native Americans (e.g., the Karitiana, >72\%), and Northeast Asians (e.g., the 317 Mongolians, >68\%), the H5 group segregates in higher frequency among Europeans (e.g., the Basques, $318>81 \%$ ), Middle Easterners (e.g., the Druze, >61\%), and some Native Americans (e.g., the Surui, >83\%) 319 (Figure 6A). Consistent with our haplotype-based PCA clustering results, we observed high frequencies 320 of the H4/H5 heterozygous form (orange in Figure 6B) across populations, with the highest found in 321 Southeast Asians and Native Americans (>60\%) and the lowest in some Melanesian and African 322 populations $(<20 \%)$.

323 Given the common presence of these two super haplogroups in humans (Figure 6A-6B), we 324 collected multi-tissue eQTL data from the GTEx Project (release v8) and found that all four tagging 
325 SNVs are TCAF2 eQTLs (Figure 6C). The H4 haplogroup (reference) alleles are significantly associated 326 with increasing TCAF2 ( $\mathrm{p}$ values $<1 \times 10^{-10}$ ) expression in thyroid and tibial nerve tissues in addition to 327 others (Figure 6C). Thus, our inferences of the differential TCAF2 expression between the H4 and H5 328 haplogroups imply possibly functional differences in the peripheral nervous system and thyroid among 329 hominin groups. Finally, we explored traits that are potentially associated with the $T C A F$ haplogroups 330 using the recently released genetic association data from the ancestry-based genetic analysis of the UK 331 Biobank (Pan-UK Biobank as of 2020.08.06; https://pan.ukbb.broadinstitute.org/). We examined SNVs

332 from the three unique diploid regions at the TCAF locus and focused on nerve- and thyroid-related traits, 333 including diabetes as thyroid dysfunctions often result in diabetes mellitus ${ }^{30}$. While in general no SNVs

334 reach genome-wide significance for traits that we interrogated, we found several suggestive association 335 signals among specific human populations. For example, we found nominal associations (uncorrected $\mathrm{p}$ 336 values < 0.05 ) for SNVS, including the four eQTLs, for hypothyroidism (phecode-244) in both African 337 and East Asian populations as well as for diabetes medication (Glimepiride) and compressed nerves in 338 samples of European descent (Figure S31) consistent with the expression pattern of these genes.

\section{DISCUSSION}

341 Genetic changes in SDs are now well recognized as an important source of functional innovation in

342 human evolution due, in part, to subsequent rounds of duplication, deletion, or inversion ${ }^{13,14}$. Such 343 regions, however, are often frequently overlooked as part of comparative studies, association studies, or 344 scans of selection because of their structural complexity and high-degree of homology. In this study, we

345 first delineate the complex structural diversity of the locus in humans and compare it to nonhuman 346 primates at the sequence level in order to reconstruct its evolutionary history ${ }^{12}$. Of note, complete 347 assembly of 15 haplotypes by tedious BAC-based sequencing was sufficient to capture more than half of 348 the genetic diversity of TCAF SDs in humans. Based on these data, we develop a model of rapid 349 diversification with respect to both haplotype and TCAF SD copy number diversity (Figures 1-3). It is 350 worth noting that despite the recent advances in long-read sequence assembly, the most complex 
351 haplotypes still remain collapsed and unassembled in some of the recently released human genomes ${ }^{31-33}$

352 (Figure S4, Table S2). We believe that this is due partly to the read-length limitation of the data as well

353 as the near-perfect sequence identity among TCAF SDs that lead to ambiguous read partitions during

354 haplotype assembly. This highlights the importance of alternate approaches, such as large-insert BAC

355 clones, to construct highly accurate haplotype-resolved assemblies. More sophisticated assembly

356 approaches and long-read technology for generating longer and even more accurate sequence reads will

357 likely make such investigations much more routine in the future ${ }^{34}$.

358 The structural complexity and copy number variation of this region underscores its mutational

359 dynamics. The high degree of sequence identity among $T C A F$ paralogs $(>99.7 \%)$, their clustered and

360 tandem orientation, and their large size $(10-60 \mathrm{kbp})$ promote recurrent structural rearrangements making

361 the entire locus genetically unstable as a result of NAHR ${ }^{13}$. Human Haplogroups 4 and 5, in particular,

362 carry more TCAF SD paralogs than others, and, thus, both are predicted to be subject to a higher

363 likelihood of structural mutation. Our analysis predicts that $\mathrm{H} 4$ would be more predisposed to structural

364 rearrangements than H5 due to a greater number of directly orientated duplications (DupA and DupB)

365 (Figure 2). This prediction is consistent with the observation of more subgroups within supergroup H4 in

366 the haplotype-based PCA (Figures S24-S27). Surprisingly, this predisposition to recurrent structural

367 diversity is specific to modern-day humans as our sampling of eight archaic hominin haplotypes suggests

368 a static H4 haplogroup without a single duplication event. Given our estimates of origin of the

369 duplications ( 1.7 Mya) and haplogroup coalescence ( 0.73 Mya), the lack of diversity in the

370 Neanderthal and Denisovan lineages is highly unlikely.

371 One of the challenges studying this region is the wide-spread IGC we identified among the TCAF

372 SDs. IGC essentially erases signatures left by previous mutation events in the acceptor site, thus biasing

373 towards younger TMRCA estimates ${ }^{35,36}$. While we mitigated such a bias by focusing our analysis on non-

374 IGC sequences, this reduced the available dataset limiting our power to infer its evolution at a finer level

375 of resolution. Interestingly, the analysis uncovered a significant correlation between paralog-specific copy

376 number and the latitudinal location of human populations precisely over a potential enhancer region, 
377 suggesting that IGC may have contributed to regulatory differences of $T C A F$ genes among human

378 populations. Such co-option of IGC has been noted before among duplicate genes. For example, a human-

379 specific, pseudogene-mediated IGC of SIGLEC11 replaced the 5' upstream region and coding exons of

380 SIGLEC11 by its adjacent pseudogene SIGLEC16P in the human lineage. This event has been

381 hypothesized as adaptive during human brain development ${ }^{37}$. While we cannot rule out the possibility of

382 confounding effects of demography resulting in the observed correlation, given the importance of $T C A F$

383 in thermal regulation and sensing, it is tempting to hypothesize that IGC contributes to local adaptation by

384 changing dosages through rapid replacement of regulatory sequences in addition to SD.

385 Our more complete reference genomes coupled with targeted cDNA sequencing also provides the

386 most comprehensive TCAF annotation to date for humans and nonhuman primates (Figures 3 and S4-

387 S12). We show that each additional TCAF SD cassette (DupA, DupB, and DupC) generates additional

388 copies of TCAF1A and TCAF2C through incomplete gene duplication. Gene duplication has been known

389 to be an important source of genetic novelty and adaptive evolution ${ }^{1,38}$, especially for the great ape

390 lineages given the surge of duplications predicted to have occurred in the hominid ancestor ${ }^{39}$. Throughout

391 the evolution of mammals, TCAF genes have been highly conserved, and human is the only lineage that is

392 known to carry additional copies. The coding sequences among the individual paralogs are highly

393 conserved as a result of their recent origin arguing for the relative importance of TCAF dosage differences

394 in regulating the TRPM8 ion channel as suggested by a previous studies ${ }^{12}$. We also observe, however,

395 considerable isoform diversity as a result of the incomplete nature of the SD particularly among TCAF2

396 paralogs suggesting a potentially more complex relationship with the ancestral genes. This pattern of

397 incomplete duplication resembles the evolution of the neural human-specific duplication SRGAP2 genes

398 , where the younger, incomplete duplicated paralog SRGAP2C inhibits the function of the ancestral copy

$399 S R G A P 2 A$, and in mice experiments this in turn prolongs the maturation of synapses and subsequently

400 increases the density of the length of synapses ${ }^{4}$. Taken together, our results bolster the hypothesis of

401 recent incomplete gene duplications and adaptive human evolution. 

same locus over the last half million years of hominin evolution. We propose that diversifying or

404 balancing selection is acting in at least some human populations, particularly out-of-African populations 405 such as Native Americans, to maintain and expand haplotype and structural diversity. While the exact 406 mechanism and phenotype that balancing selection are acting upon are not clear, the differential eQTL 407 signal related to TCAF2 expression between supergroup H4 and H5 alleles in multiple tissues would be 408 consistent with antagonistic pleiotropy, where two haplotypes have opposing effects on two different 409 traits depending on either environmental or life-history conditions ${ }^{40,41}$. In contrast, Neanderthal and 410 Denisovan show a paucity of genetic variation, and while the sample size is still limited, this observation 411 is unlikely to change with the sequencing of additional archaic genomes. Moreover, both the significantly 412 negative Tajima's $D$ statistic and the single-haplotype without duplication are highly unlikely observed 413 under neutrality after accounting for differences explained by demographic history. Our inference results, 414 thus, strongly argue that positive selection has reduced genetic diversity at the TCAF locus in these 415 archaic hominin lineages.

416 The opposing effects on the TCAF2 expression found between $\mathrm{H} 4$ and H5 haplogroups in the 417 thyroid is particularly intriguing. Thyroid activity is one of the most important determinants of overall 418 energy expenditure and basal metabolic rate in human body ${ }^{42}$. Not only does the thyroid hormone 419 maintain core body temperature when exposed to cold, but it also regulates metabolism through action in 420 the brain, brown fat, skeletal muscle, etc. ${ }^{43}$. Thyroid dysfunctions, both hyperthyroidism and

421 hypothyroidism, often lead to metabolic disorders, such as diabetes mellitus ${ }^{30}$, and also cause neurological 422 disorders during fetal and brain development due to low levels of maternal thyroid hormones from 423 maternal hypothyroidism or lack of iodine in diet ${ }^{44,45}$. Despite no genome-wide significant genetic 424 associations, we did identify suggestive trends between specifically those eQTL variants and 425 hypothyroidism, diabetes medication, as well as pinched nerves. Interestingly, the TRPM8 ion channel, in 426 addition to its role in detecting environmental cold, is also a known regulator of insulin homeostasis

427 through neuronal control of liver insulin clearance and, thus, required for precise thermoregulatory 
428 responses to cold and fasting ${ }^{46,47}$. In addition, a rare variant within the $T C A F$ locus (chr7:143735931) was

429 recently reported to be associated with type 2 diabetes in sub-Saharan African populations ${ }^{48}$. Given this

430 variant's restricted geographic distribution and low frequency in the GTEx data (allele frequency: 0.4\%;

431 GTEx portal as of 2020.07.21), it explains neither the observed patterns of diversity nor the selection and

432 the eQTL signals observed here. Therefore, if the TCAF haplotype and structural diversity affect an

433 individual's ability to regulate TRPM8 ion-channel gating and, thus, body temperature, it is possible that

434 the antagonistic forces of selection promote adaptations to a cold environment or an enhanced ability to

435 dynamically adapt to changing conditions, such as diets.

436 While it is still controversial, cold weather is thought to have forced Neanderthals to move from

437 coastal areas to further inland, where they would have had to adapt to iodine-poor diets, which may in

438 turn have affected brain development in this species ${ }^{44,49}$. Iodine deficiency is one of the common causes

439 of hypothyroidism (reduced thyroid hormone levels), which is characterized by lower core body

440 temperature and decreased resting energy expenditure ${ }^{30}$. Despite the limited understanding of the

441 molecular bases underlying the interactions between $T C A F$ and thyroid hormones, one possibility may be

442 that selection on $\mathrm{H} 4$ increased TCAF2 expression and subsequently suppressed the activity of TRPM8 in

443 the thyroid of archaic hominins. This may have improved physiologically adaptation to cold weather and

444 low iodine-intake diets.

445

\section{METHODS}

447 Copy number genotyping in high-coverage Illumina short-read data

448 To explore the temporal and spatial copy number variation of TCAF SDs, we leveraged a large collection

449 of publicly available high-coverage Illumina genomes from 1,102 modern human samples, four archaic

450 hominins, and 71 nonhuman great apes ${ }^{17-23}$. In short, sequencing reads were divided into multiples of $36-$

451 mer and then mapped to a repeat-masked human reference genome (GRCh38) using mrsFAST (v3.4.1) ${ }^{50}$.

452 We allowed up to two mismatches per alignment to increase our mapping sensitivity and corrected for

453 possible biases in read depth due to different levels of GC content in sequencing reads. Finally, the copy 
454 number estimate was computed by summarizing over all mappable bases at the locus of interest for each

455 sample.

457 Library processing and assembly for TCAF BAC haplotypes

458 We obtained the human BAC libraries used in this study from the Virginia Mason Research Center and

459 the nonhuman primate ones from the Children's Hospital Oakland Research Institute. Probes for regions

460 of interest were designed, radioactively labeled and hybridized to the Performa filters, washed, exposed to

461 Phosphor screens, and scanned on a Typhoon scanner. Positives are called and corresponding clones

462 selected from the BAC library. We prepared barcoded libraries from clone DNA using Illumina-

463 compatible Nextera DNA sample prep kits (Epicentre, catalog number GA09115) as described

464 previously ${ }^{51}$ and carried out paired-end sequencing (125 bp reads) on an Illumina HiSeq 2500. Reads

465 were then mapped to the reference genome, GRCh38, to identify singly unique nucleotide k-mers

466 (SUNKs) ${ }^{16}$. Non-overlapping BACs were pooled and sheared as described previously ${ }^{52}$. Libraries

467 were processed using the PacBio SMRTbell Template Prep kit following the protocol "Procedure and

468 Checklist — 20 kb Template Preparation Using BluePippin Size-Selection System” with the addition of

469 barcoded adaptors during ligation. Up to ten barcoded libraries were then pooled at equimolar

470 amounts and size-selected as a pool on the Sage PippinHT with a start value of 10,000-12,000 and an

471 end value of 50,000. The resulting library was then sequenced on one Sequel SMRT cell 1M by

472 diffusion using Sequel v3.0 chemistry. We performed de novo assembly of pooled BAC inserts using

473 Canu (v1.5). Reads were masked for vector sequence (pCC1BAC) and assembled with Canu, then

474 subjected to consensus sequence calling with Arrow

475 (https://github.com/PacificBiosciences/GenomicConsensus). We reviewed PacBio assemblies for

476 misassembly by visualizing the read depth of PacBio reads in Parasight (v7.6,

477 http://baileylab.brown.edu/parasight/download.html), using coverage summaries generated during the

478 resequencing protocol. We performed BLAST (v2.10.1) pairwise alignment between the BAC sequences

479 for the construction of haplotypes. Complete contigs for $15 T C A F$ haplotypes must satisfy the following 
two conditions: (1) overlapped DNA segment between BAC sequences must be at least $99.99 \%$ identical

481 with no tolerated mismatch and (2) contig must anchor to unique location on both ends when mapping

482 back to the human genome reference GRCh38.

483

484 Identification of haplogroups and interlocus gene conversion (IGC) tracts

485 All sequence alignments in this study were performed using MAFFT (v7.453). To determine the

486 structural differences among the haplogroups, we built single-base level sequence identity profiles using

487 pairwise sequence alignment, followed by smoothing over windows of $500 \mathrm{bp}$ with a step size of $100 \mathrm{bp}$.

488 Because we are interested in recent, large IGC events, empirical IGC tracts are determined as more than

489 two consecutive windows that have $100 \%$ sequence identity. To find additional support, we use a model-

490 based program, GENECONV (v1.81a), to identify pairs of uninterrupted sequences with 100\% sequence

491 identity that are longer than expected given the overall pattern of variable sites in an alignment. To be

492 conservative, we considered IGC tracts that have both simulation- and Karlin-Altschul- $\mathrm{p}$ values $<0.05$

493 reported by GENECONV.

495 Full-length non-chimeric (FLNC) transcripts for TCAF genes using long-read cDNA sequencing

496 Total RNA was harvested from six human tissues (dorsal root ganglion: Clontech Laboratories, Inc.,

497 Takara [catalog \#636150]; esophagus: Clontech Laboratories, Inc., Takara [catalog \#636178]; fibroblast

498 (Coriell); skin: Biochain Institute Inc. [catalog \#R1234218-P], fetal brain: NCBI BioSample:

499 SAMN09459150; testis: Clontech Laboratories, Inc., Takara [catalog \#636533]) and one chimpanzee

500 lymphoblast cell line. We purified polyA RNA using oligo-dT magnetic beads (Dynal: Thermo Fisher

501 Scientific Inc.). Double-stranded cDNA with 96 random barcodes was prepared, amplified and subjected

502 to hybridization capture followed the protocols detailed in (Dougherty et al. 2018). Hybridization probes

503 were designed to target the duplicated exons of TCAF2, and sequences are given in Supplemental Table

504 S2b of Dougherty et al., 2018. 
Following post-capture PCR, the amplified dsDNA was purified on magnetic beads (AMPure PB;

506 PacBio) and then subjected to library preparation for long-read sequencing (SMRTbell Template Prep Kit

507 1.0; PacBio with barcoded SMRTbell adapters). SMRT sequencing was performed on the Sequel

508 platform with Sequel v3.0 chemistry (PacBio) with LR SMRT Cells with 2-hour pre-extension and 20-

509 hour movies. Reads corresponding to each sample were extracted by their SMRTbell barcodes and

510 circular consensus sequences were generated from the raw subreads using SMRT Link with minimum

511 number of pass set to 1 . The program lima in the Iso-Seq3 pipeline

512 (https://github.com/PacificBiosciences/IsoSeq3) was applied to remove the 5' and 3' dual barcodes and

513 also obtain the unclustered FLNC reads. Parameters used were: lima --isoseq --dump-clips. We did not

514 cluster the FLNC reads further because highly identical paralogous transcripts could undesirably cluster

515 together in this step. Due to the variability of the yields of FLNC transcripts across samples and loci, we

516 used data from various combinations of samples in subsequent analyses when applicable.

517 To determine the coding potential for each FLNC transcript, we use the program ANGEL

518 (https://github.com/PacificBiosciences/ANGEL) to call open reading frames with a minimum length

519 cutoff of 200 amino acids and proper stop codons. All transcripts with open reading frames were mapped

520 to the seven TCAF haplogroups (Tables S4 and S5) using minimap2 (v2.17-r941) and individual best

521 placements to specific haplogroups were determined using sequence identity. Given the recent history of

522 the TCAF duplications, we required a $>99 \%$ sequence identity for an alignment to be considered a match

523 between an FLNC read and the haplogroup contig and most have at least an overlap of 200 bases. To

524 improve the sensitivity of FLNC read assignments, we allowed multi-mapping placements for an FLNC

$525 \mathrm{read}$ as long as the difference in sequence identity between alignments is $<0.01 \%$. Finally, to determine

526 gene models and isoforms in each haplogroup, we focused our analysis on FLNC transcripts with more

527 than 10 reads (Table S5).

528 
529 Single-nucleotide variant (SNV) calling for unique diploid sequences at TCAF locus

530 To perform population genetic analyses, we generated a joint call set for 840 published high-coverage

531 short-read Illumina genomes, including 828 Human Genome Diversity Project (HGDP) samples, eight

532 chimpanzee samples, and the four high-coverage archaic genomes. Because the current human reference

533 GRCh38 has a gap at the TCAF locus, we constructed two custom references for chromosome 7: one with

534 Haplogroup 4 and the other with Haplogroup 5, in additional to a TCAF-SDs-hardmasked

535 (chr7:143521769-143874696, GRCh38) chromosome 7. Paired-end data were aligned to the two custom

536 references using BWA-MEM (v0.7.12), while ancient DNA data were mapped using BWA (v0.7.17) with

537 parameters with parameters: $-\mathrm{n} 0.01-\mathrm{o} 2-116500{ }^{19}$. SNV genotypes were jointly generated using

538 haplotype caller FreeBayes (v1.0.2-6-g3ce827d) with the following parameters: “--min-coverage 10 --

539 use-best-n-alleles 4”. To ensure genotype quality, we excluded variants that were found within $10 \mathrm{bp}$ of

540 indels and have quality score $($ QUAL) $<20$. We identified a total of 1,275 and 1,295 SNVs from the call

541 sets of Haplogroups 4 and 5, respectively, among the 840 samples. Note that we excluded 38 and 30

542 samples from the call sets of Haplogroups 4 and 5, respectively, due to $>10 \%$ missing data to ensure the

543 quality of genotype calls and downstream analyses. Because the results and interpretations of all

544 downstream analyses are highly compatible, we concluded no obvious reference biases between the two

545 sets. Unless otherwise stated, we reported results from the Haplogroup 4 call set because it has slightly

546 better mapping quality.

547

$548 \quad \underline{\text { Phylogenetic analyses }}$

549 To infer the phylogenetic relationships for TCAF SDs and the unique diploid sequences in primates, we 550 performed both maximum likelihood (IQ-TREE, v1.6.11) 53 and Bayesian phylogenetic-based (BEAST 551 v2.5.0) ${ }^{54}$ analyses. First, we determined parameters and models using ModelGenerator (v0.85) ${ }^{55}$ and

552 compared with the recommendations from the model-selection feature of IQ-TREE (using the parameters:

553 “-bb 1000 -redo -nt 1 -m MFPMERGE”). Based on the best-fit model recommended, to run BEAST, in

554 general we set 1) GAMMA Category Count $=5$, shape parameter $=0.1$, Proportion Invariant $=0.38$, and 
555 using the GTR substitution model (all rates = 1.0) for the Site Model, and 2) Relaxed Clock Log Normal

556 over branches on the tree for Clock Model. For tree priors, we used Calibrated Yule Model, and while

557 kept most of the parameters of the priors as default, for birthRate and clockRate we used Gamma(0.001,

558 1000) and set calibrations based on human-chimpanzee and human-rhesus macaque divergence

559 following the distributions of a $\log$-normal $(M=6500000, S=0.09)$ and $\log$-normal $(M=10000000$,

$560 \mathrm{~S}=0.09)$, respectively. For the analysis involving the inferred archaic hominin haplotypes, we also set

561 dates for terminal samples to account for the differences between contemporary and fossil samples. For

562 each run, we drew a date (in years) from a uniform [36000, 100000] for the Neanderthals and a uniform

563 [50000, 80000]. For each locus, we performed five independent runs to infer the phylogeny using a chain

564 length of 50,000,000 samples and recorded every 1,000 samples. We used the accompany program Tracer

565 (v.1.7.1) to determine the quality of each run and, in general, we used the first $10 \%$ as burn-in and only

566 kept runs with ESS > 500. All phylogenetic trees were plotted using Figtree (v1.4.3).

567

568 Population genetic analyses

569 All population genetic analyses in this study were based on SNVs from the three unique diploid regions at

570 the TCAF locus (chr7:143,501,000-143,521,000, chr7:143,729,525-143,741,525, chr7:143,875,000-

571 143,895,000 [GRCh38]) using the HGDP, archaic hominin, and chimpanzee samples. Haplotypes were

572 inferred by applying BEAGLE (v5.1-25Nov19.28d) with default settings to modern human, archaic

573 hominin, and chimpanzee samples separately. We explored the diversity of the TCAF haplotype using a

574 haplotype-based PCA to classify and assign haplotypes into individual clusters. Cluster assignments were

575 based on the supervised k-means algorithm using the top $\mathrm{N}$ informative PCs, where $\sum_{i=1}^{N} \operatorname{Var}\left(P C_{i}\right)>$

576 0.9. To determine the best number of cluster $\mathrm{k}$, for each possible $\mathrm{k}$ between 1 and 20, we computed the

577 within groups sum of squares (WSS) using a distance matrix among the top N PCs and determined the

578 best $\mathrm{k}$ when $W S S_{k+1}-W S S_{k}<$ delta, where delta was chosen as 500 according to our analysis. Data

579 visualization of the haplotype clustering was performed using the machine learning technique t-

580 distributed stochastic neighbor embedding (t-SNE) ${ }^{28}$ implemented in the R package Rtsne 
581 (https://github.com/jkrijthe/Rtsne) using the following parameters: “perplexity=50, max_iter=5000, 582 early_exag_coeff $=12$, exaggeration $=4$, stop_lying_iter $=1000$, check_duplicates $=$ FALSE, dims $=3$ ". We 583 optimized the t-SNE analysis by running five replicates of this computation and identifying the smallest

584 Kullback-Leibler distance as the candidate for final visualization ${ }^{28}$.

585 To test for signals of natural selection, we computed the Tajima's $D$ statistic ${ }^{56}$ over 2000 bp

586 windows over the $52.3 \mathrm{kbp}$ unique diploid sequences for individual populations as well as the archaic

587 samples. Because the Tajima's D statistic is sensitive to population history, we performed a large

588 coalescent simulation using $\mathrm{MaCS}^{57}$ and 1,000 demographic models as described previously ${ }^{25,58}$ to

589 account for past histories between modern and archaic hominins and among multiple African and non-

590 African populations. Note that our simulations carefully match the local mutation rate and recombination

591 rate variation at these regions to avoid possible biases to our selection inferences. To estimate the

592 TMRCA for a locus of interest, we used the Thomson's estimator ${ }^{59}$. LD measurements among SNVs

593 were computed using Lewontin's $\mathrm{D}^{\prime 60}$ and $\mathrm{R}^{2}$ implemented in PLINK (v1.09, www.cog-

594 genomics.org/plink/1.9/) ${ }^{61}$. Note only SNVs with minor allele frequencies $>10 \%$ were included for this 595 analysis because lower frequency variants are less informative about linkage.

\section{ACKNOWLEDGEMENTS}

598 The authors thank T. Brown for assistance in editing this manuscript. We also thank S.C. Murali and D.S.

599 Gordon for help submitting data to the NCBI database. Funding: This work was supported, in part, by

600 the US National Institutes of Health (NIH) grant R01HG002385 to E.E.E. P.H. is supported by the NIH

601 Pathway to Independence Award (NHGRI, K99HG011041). S.C. was supported by a National Health and

602 Medical Research Council (NHMRC) C. J. Martin Biomedical Fellowship (1073726). E.E.E. is an

603 investigator of the Howard Hughes Medical Institute. Author contributions: P.H. and E.E.E. designed

604 and planned experiments. V.D., C.B., S.C., A.P.L., K.M.M., M.S., A.E.W., and J.G.U. prepared libraries

605 and generated and analyzed sequencing data. P.H., V.D., M.R.V., and T.H. performed variant calling and

606 bioinformatics analyses. P.H., V.D., M.R.V., and P.C.D. analyzed long-read sequencing data and 

assembled contigs. P.H. and Y.M. performed population genetic and phylogenetic inferences. A.P.L., K.M.M., P.C.D., and J.G.U. generated Iso-Seq transcript data. P.H. and E.E.E. wrote the manuscript. Declaration of interests: J.G.U. is an employee of Pacific Biosciences, Inc.

610

DATA AVAILABILITY: All data used in this study, including assembled BAC contigs and Iso-Seq capture transcript data, are deposited in NCBI under the BioProject: PRJNA657884, BioSample:

SAMN09459150; these data are available to anyone for purposes of reproducing or extending the

614 analysis.

\section{REFERENCES}

618 1. Ohno, S. Evolution by gene duplication, xv, 160 p. (Springer-Verlag, New York, 1970).

619 2. Kimura, M. \& Ohta, T. On some principles governing molecular evolution. Proc Natl Acad Sci U S A 71, 2848-52 (1974).

621 3. Boyd, J.L. et al. Human-chimpanzee differences in a FZD8 enhancer alter cell-cycle dynamics in the developing neocortex. Curr Biol 25, 772-779 (2015).

4. Charrier, C. et al. Inhibition of SRGAP2 function by its human-specific paralogs induces neoteny during spine maturation. Cell 149, 923-35 (2012).

5. Dennis, M.Y. et al. Evolution of human-specific neural SRGAP2 genes by incomplete segmental duplication. Cell 149, 912-22 (2012).

6. Florio, M. et al. Human-specific gene ARHGAP11B promotes basal progenitor

amplification and neocortex expansion. Science 347, 1465-70 (2015).

7. Fiddes, I.T. et al. Human-Specific NOTCH2NL Genes Affect Notch Signaling and Cortical Neurogenesis. Cell 173, 1356-1369 e22 (2018).

8. Dennis, M.Y. \& Eichler, E.E. Human adaptation and evolution by segmental duplication. Curr Opin Genet Dev 41, 44-52 (2016).

9. Nuttle, X. et al. Emergence of a Homo sapiens-specific gene family and chromosome 16p11.2 CNV susceptibility. Nature 536, 205-9 (2016).

10. Colburn, R.W. et al. Attenuated cold sensitivity in TRPM8 null mice. Neuron 54, 379-86 (2007).

11. Key, F.M. et al. Human local adaptation of the TRPM8 cold receptor along a latitudinal cline. PLoS Genet 14, e1007298 (2018).

12. Gkika, D. et al. TRP channel-associated factors are a novel protein family that regulates TRPM8 trafficking and activity. J Cell Biol 208, 89-107 (2015).

13. Eichler, E.E. Recent duplication, domain accretion and the dynamic mutation of the human genome. Trends Genet 17, 661-9 (2001).

14. Chaisson, M.J., Wilson, R.K. \& Eichler, E.E. Genetic variation and the de novo assembly of human genomes. Nat Rev Genet 16, 627-40 (2015). 
15. Chaisson, M.J.P. et al. Multi-platform discovery of haplotype-resolved structural variation in human genomes. Nat Commun 10, 1784 (2019).

16. Sudmant, P.H. et al. Diversity of human copy number variation and multicopy genes. Science 330, 641-6 (2010).

17. Meyer, M. et al. A high-coverage genome sequence from an archaic Denisovan individual. Science 338, 222-6 (2012).

18. Prado-Martinez, J. et al. Great ape genetic diversity and population history. Nature 499, 471-5 (2013).

19. Prufer, K. et al. A high-coverage Neandertal genome from Vindija Cave in Croatia. Science 358, 655-658 (2017).

20. Prufer, K. et al. The complete genome sequence of a Neanderthal from the Altai Mountains. Nature 505, 43-9 (2014).

21. Mafessoni, F. et al. A high-coverage Neandertal genome from Chagyrskaya Cave. Proc Natl Acad Sci U S A 117, 15132-15136 (2020).

22. Bergstrom, A. et al. Insights into human genetic variation and population history from 929 diverse genomes. Science 367(2020).

23. Mallick, S. et al. The Simons Genome Diversity Project: 300 genomes from 142 diverse populations. Nature 538, 201-206 (2016).

24. Redon, R. et al. Global variation in copy number in the human genome. Nature 444, 44454 (2006).

25. Hsieh, P. et al. Adaptive archaic introgression of copy number variants and the discovery of previously unknown human genes. Science 366(2019).

26. Magness, C.L. et al. Analysis of the Macaca mulatta transcriptome and the sequence divergence between Macaca and human. Genome Biol 6, R60 (2005).

27. Kronenberg, Z.N. et al. High-resolution comparative analysis of great ape genomes. Science 360(2018).

28. Maaten, L.v.d. \& Hinton, G. Visualizing data using t-SNE. Journal of machine learning research 9, 2579-2605 (2008).

29. Stephens, J.C. et al. Haplotype variation and linkage disequilibrium in 313 human genes. Science 293, 489-93 (2001).

30. Biondi, B., Kahaly, G.J. \& Robertson, R.P. Thyroid Dysfunction and Diabetes Mellitus: Two Closely Associated Disorders. Endocr Rev 40, 789-824 (2019).

31. Nurk, S. et al. HiCanu: accurate assembly of segmental duplications, satellites, and allelic variants from high-fidelity long reads. bioRxiv, 2020.03.14.992248 (2020).

32. Garg, S. et al. Accurate chromosome-scale haplotype-resolved assembly of human genomes. bioRxiv, 810341 (2020).

33. Porubsky, D. et al. A fully phased accurate assembly of an individual human genome. bioRxiv, 855049 (2019).

34. Vollger, M.R. et al. Improved assembly and variant detection of a haploid human genome using single-molecule, high-fidelity long reads. Ann Hum Genet 84, 125-140 (2020).

35. Chen, J.M., Cooper, D.N., Chuzhanova, N., Ferec, C. \& Patrinos, G.P. Gene conversion: mechanisms, evolution and human disease. Nat Rev Genet 8, 762-75 (2007).

36. Osada, N. \& Innan, H. Duplication and gene conversion in the Drosophila melanogaster genome. PLoS Genet 4, e1000305 (2008).

37. Hayakawa, T. et al. A human-specific gene in microglia. Science 309, 1693 (2005). 
691 38. Lynch, M. \& Katju, V. The altered evolutionary trajectories of gene duplicates. Trends

692 Genet 20, 544-9 (2004).

693 39. Marques-Bonet, T., Girirajan, S. \& Eichler, E.E. The origins and impact of primate

694

695

696 segmental duplications. Trends Genet 25, 443-54 (2009).

40. Faria, R., Johannesson, K., Butlin, R.K. \& Westram, A.M. Evolving Inversions. Trends Ecol Evol 34, 239-248 (2019).

41. Merot, C., Llaurens, V., Normandeau, E., Bernatchez, L. \& Wellenreuther, M. Balancing selection via life-history trade-offs maintains an inversion polymorphism in a seaweed fly. Nat Commun 11, 670 (2020).

42. Kim, B. Thyroid hormone as a determinant of energy expenditure and the basal metabolic rate. Thyroid 18, 141-4 (2008).

43. Mullur, R., Liu, Y.Y. \& Brent, G.A. Thyroid hormone regulation of metabolism. Physiol $\operatorname{Rev}$ 94, 355-82 (2014).

44. Stenzel, D. \& Huttner, W.B. Role of maternal thyroid hormones in the developing neocortex and during human evolution. Front Neuroanat 7, 19 (2013).

45. Forhead, A.J. \& Fowden, A.L. Thyroid hormones in fetal growth and prepartum maturation. J Endocrinol 221, R87-R103 (2014).

46. McCoy, D.D. et al. Enhanced insulin clearance in mice lacking TRPM8 channels. Am J Physiol Endocrinol Metab 305, E78-88 (2013).

47. Reimundez, A. et al. Deletion of the Cold Thermoreceptor TRPM8 Increases Heat Loss and Food Intake Leading to Reduced Body Temperature and Obesity in Mice. J Neurosci 38, 3643-3656 (2018).

48. Chen, J. et al. Genome-wide association study of type 2 diabetes in Africa. Diabetologia 62, 1204-1211 (2019).

49. Dobson, J.E. The iodine factor in health and evolution. Geographical Review 88, 3-28 (1998).

50. Hach, F. et al. mrsFAST: a cache-oblivious algorithm for short-read mapping. Nat Methods 7, 576-7 (2010).

51. Steinberg, K.M. et al. Structural diversity and African origin of the 17q21.31 inversion polymorphism. Nat Genet 44, 872-80 (2012).

52. Vollger, M.R. et al. Long-read sequence and assembly of segmental duplications. Nat Methods 16, 88-94 (2019).

53. Kalyaanamoorthy, S., Minh, B.Q., Wong, T.K.F., von Haeseler, A. \& Jermiin, L.S. ModelFinder: fast model selection for accurate phylogenetic estimates. Nat Methods 14, 587-589 (2017).

54. Bouckaert, R. et al. BEAST 2: a software platform for Bayesian evolutionary analysis. PLoS Comput Biol 10, e1003537 (2014).

55. Keane, T.M., Creevey, C.J., Pentony, M.M., Naughton, T.J. \& McLnerney, J.O. Assessment of methods for amino acid matrix selection and their use on empirical data shows that ad hoc assumptions for choice of matrix are not justified. BMC Evol Biol 6, 29 (2006).

56. Tajima, F. Statistical method for testing the neutral mutation hypothesis by DNA polymorphism. Genetics 123, 585-95 (1989).

57. Chen, G.K., Marjoram, P. \& Wall, J.D. Fast and flexible simulation of DNA sequence data. Genome Res 19, 136-42 (2009). 
736

737

738

739

740

741

742

743

744

745

746
58. Gutenkunst, R.N., Hernandez, R.D., Williamson, S.H. \& Bustamante, C.D. Inferring the joint demographic history of multiple populations from multidimensional SNP frequency data. PLoS Genet 5, e1000695 (2009).

59. Thomson, R., Pritchard, J.K., Shen, P., Oefner, P.J. \& Feldman, M.W. Recent common ancestry of human Y chromosomes: evidence from DNA sequence data. Proc Natl Acad Sci U S A 97, 7360-5 (2000).

60. Lewontin, R.C. The Interaction of Selection and Linkage. I. General Considerations; Heterotic Models. Genetics 49, 49-67 (1964).

61. Chang, C.C. et al. Second-generation PLINK: rising to the challenge of larger and richer datasets. Gigascience 4, 7 (2015). 
Table 1. Summary of 15 assembled TCAF haplotypes constructed using large-insert BAC libraries and long-read sequencing. BAC clones were selected and sequenced using the PacBio long-read sequencing technology and assembled into individual haplotypes (Methods). Copy number of TCAF segmental duplication (SD) cassettes and the classification for individual haplotypes were determined by Miropeats and sequence alignment analysis (Figures 2 and S5-S13).

\begin{tabular}{llccccc}
\hline Haplotype ID & $\begin{array}{l}\text { BAC library } \\
\text { (species or population) }\end{array}$ & $\begin{array}{c}\text { Length } \\
\text { (bp) }\end{array}$ & $\begin{array}{c}\text { Length of } \text { TCAF } \\
\text { SD cassettes (bp) }\end{array}$ & $\begin{array}{c}\text { Copy number of } \\
\text { TCAF SD cassettes }\end{array}$ & \%GC & Haplogroup \\
\hline CHM1 & CHM1 & 368,013 & 277,806 & 2 & 39.83 & Haplogroup 2-1 \\
\hline VMRC53_hapA & NA12878 (European) & 433,048 & 277,806 & 2 & 39.56 & Haplogroup 2-2 \\
\hline VMRC53_hapB & NA12878 (European) & 425,306 & 273,483 & 2 & 39.63 & Haplogroup 3-2 \\
\hline VMRC61_hapA & HG00732 (Puerto Rican) & 337,690 & 277,856 & 2 & 39.91 & Haplogroup 2-2 \\
\hline VMRC61_hapB & HG00732 (Puerto Rican) & 435,583 & 405,366 & 3 & 39.71 & Haplogroup 4 \\
\hline VMRC62_hapA & HG00733 (Puerto Rican) & 395,405 & 277,854 & 2 & 39.60 & Haplogroup 2-2 \\
\hline VMRC64_hapA & NA19240 (Yoruba) & 323,367 & 260,853 & 2 & 39.94 & Haplogroup 2-2 \\
\hline VMRC64_hapB & NA19240 (Yoruba) & 348,654 & 277,808 & 2 & 40.07 & Haplogroup 2-2 \\
\hline VMRC66_hapA & NA19434 (Luhya) & 496,357 & 406,131 & 3 & 40.00 & Haplogroup 5 \\
\hline VMRC69_hapA & HG00514 (Han Chinese) & 387,079 & 277,712 & 2 & 39.29 & Haplogroup 3-1 \\
\hline VMRC73_hapA & GM10539 (Melanesian) & 247,628 & 145,427 & 1 & 39.93 & Haplogroup 1 \\
\hline VMRC73_hapB & GM10539 (Melanesian) & 222,558 & 145,424 & 1 & 39.85 & Haplogroup 1 \\
\hline CH251_contig & CH251 (Pan troglodytes) & 273,442 & 127,988 & 1 & 39.90 & Ancestral-like \\
\hline CH277_contig & CH277 (Gorilla gorilla) & 241,956 & 140,234 & 1 & 40.98 & Ancestral-like \\
\hline CH250_contig & CH250 (Rhesus macaque) & 225,909 & 140,184 & Ancestral-like \\
\hline
\end{tabular}




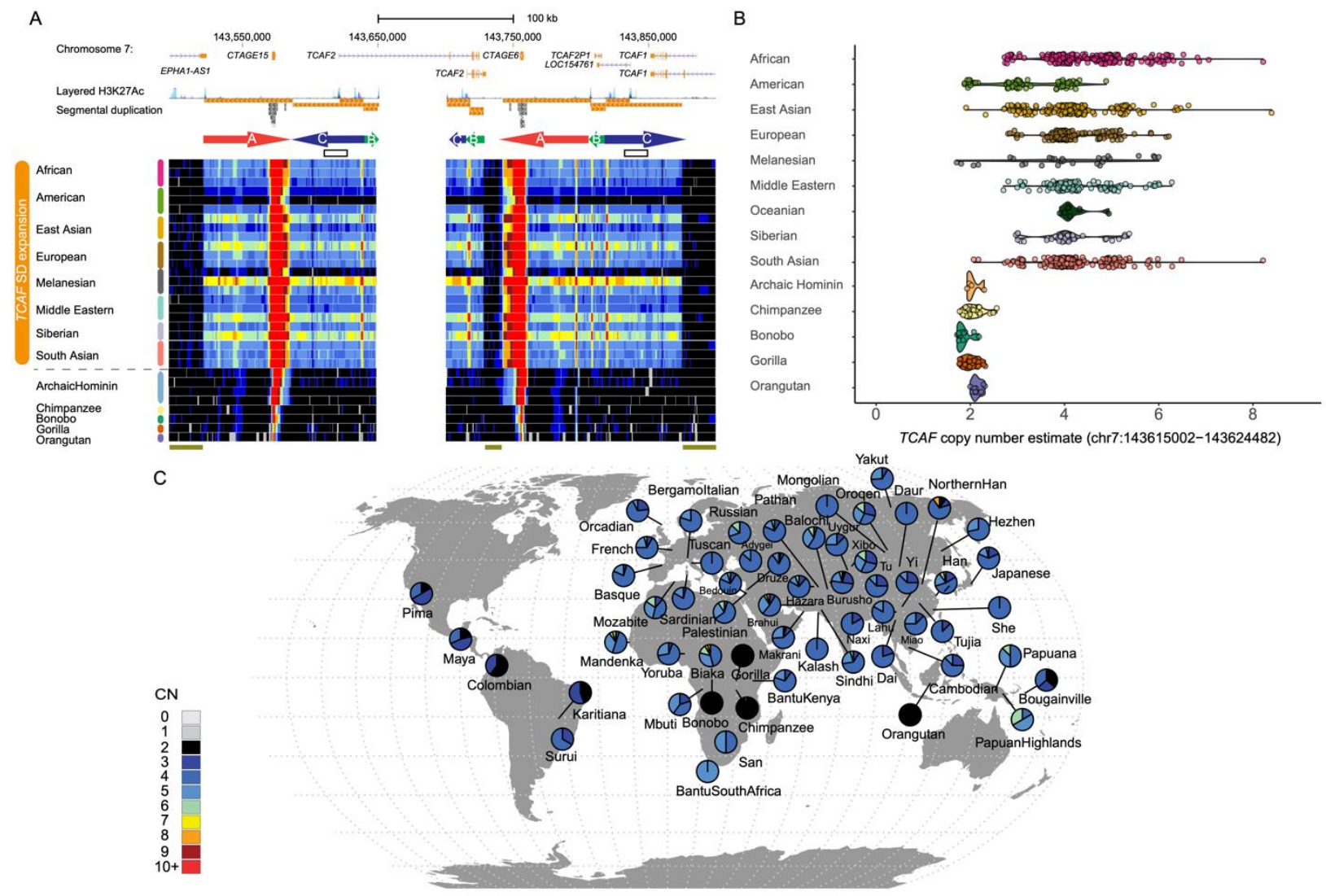

Figure 1. Copy number variation of TCAF SDs in a collection of diverse human and nonhuman samples. Copy numbers were estimated using read-depth based genotyping method. (A) Copy number $(\mathrm{CN})$ heat maps, where each row represents the $\mathrm{CN}$ of a sample over the TCAF locus. The colored arrows $(\mathrm{A}, \mathrm{B}$, and $\mathrm{C}$ ) represent the three major SD blocks in this region. The white area in the middle represents the gap present in the human reference genome (GRCh38). The two white boxes show a putative interlocus gene conversion event that correlates with latitudinal locations of populations (Figure S16S18). Dark green bars at the bottom indicate the unique diploid sequences used for downstream phylogenetic and population genetic analyses (chr7:143,501,000-143,521,000, chr7:143,729,525143,741,525, chr7:143,875,000-143,895,000 [GRCh38]). (B) Distributions of the overall TCAF CN genotypes among samples from nonhuman great apes, archaic hominins, and modern humans using a representative region (chr7:143,615,002-143,624,482). (C) Geographic distribution of the overall TCAF $\mathrm{CN}$ genotypes in the 54 Human Genome Diversity Project (HGDP) populations and the nonhuman great ape samples. Geo-coordinates for the populations are listed in Table S8. Pie charts shows the CN frequency distribution for a given population (colors correspond to those in the $\mathrm{CN}$ heat maps). 

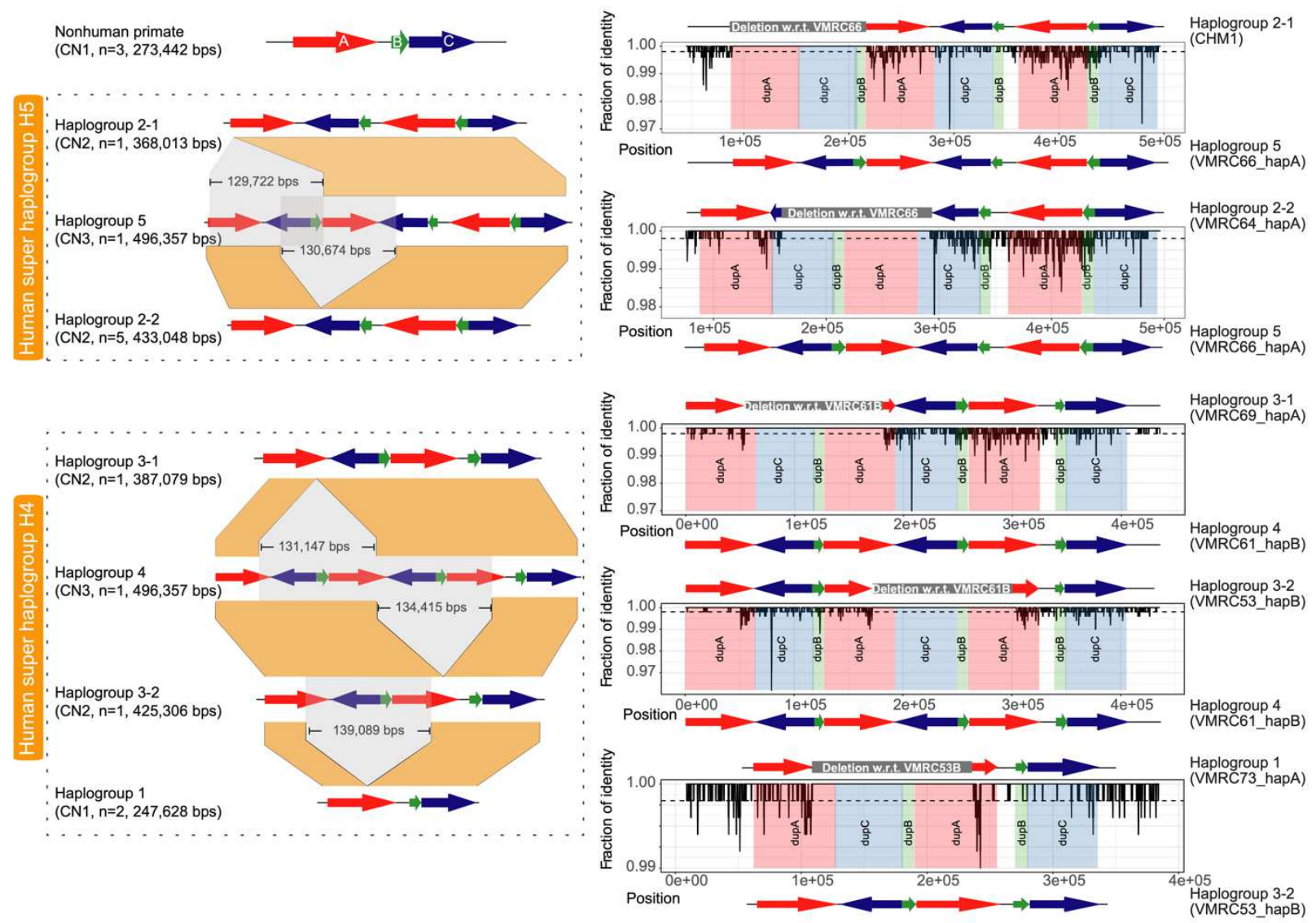

Figure 2. Complex structural haplotype diversity at the 7q35 TCAF locus in humans. Left panel: Schematic representations of the major- (super haplogroups $\mathrm{H} 4$ and $\mathrm{H} 5$ ) and sub-haplogroups identified from 15 targeted BAC long-read assemblies. Colored arrows are TCAF SDs, and the gray and orange areas indicate putative deletion events and orthologous sequences between haplogroups. For example, despite the similarity in structure, Haplogroup 2-1 and Haplogroup 2-2 are likely resulted from different deletion events from Haplogroup 5 based on comparative sequence data. Right panel: The comparative sequence analysis between individual haplogroups shows pairwise sequence identities (black lines) over $500 \mathrm{bp}$ windows (sliding by $100 \mathrm{bp}$ ). With each plot, colored rectangles correspond to TCAF SDs, while the white region embedded between a DupA (red) and a DupB (green) blocks represents a $12.3 \mathrm{kbp}$ single-copy unique sequence. Gray rectangles above each sequence identity plots indicate the locations of putative structure variants (Table S3). 


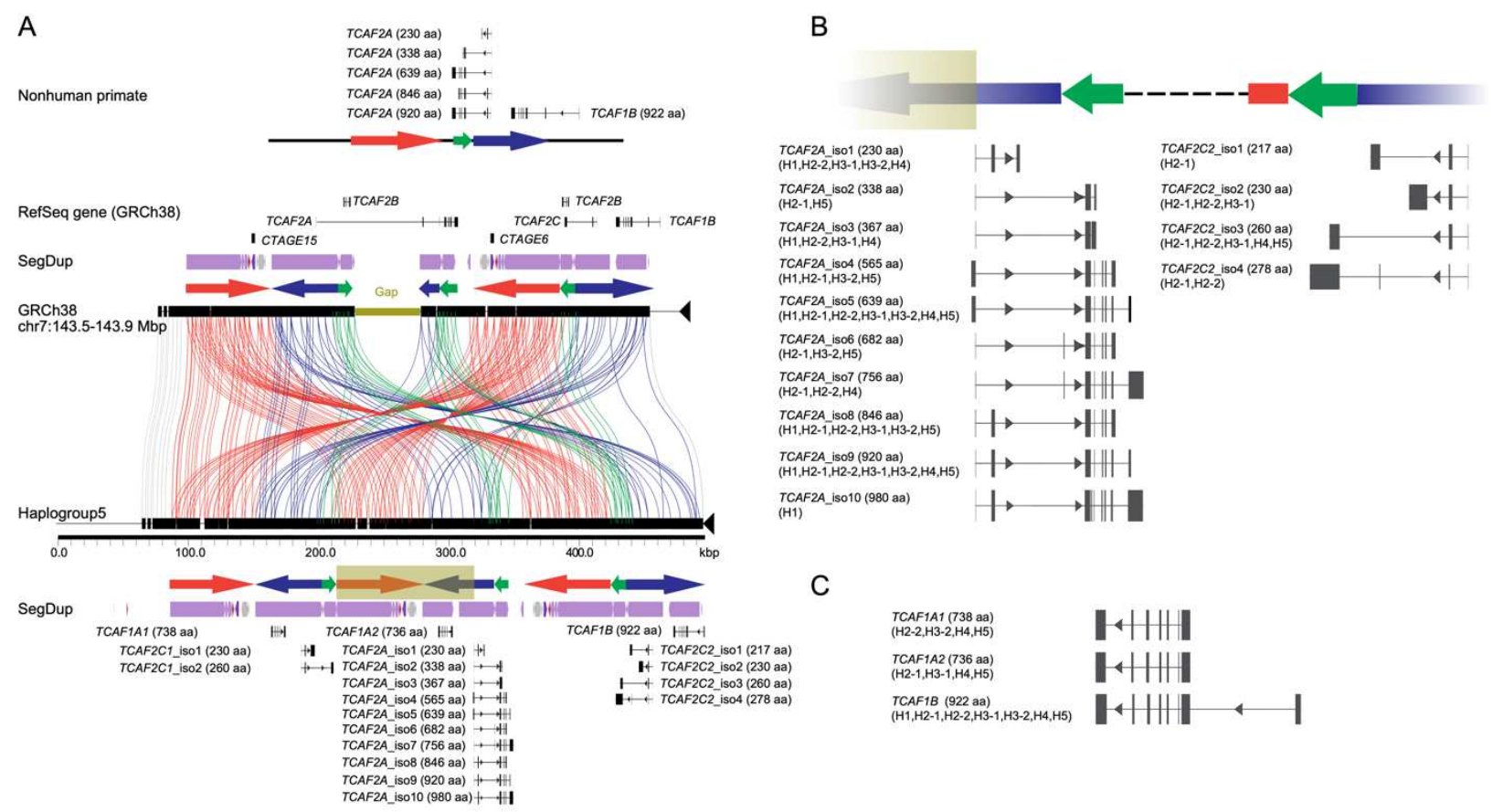

Figure 3. TCAF gene and isoform diversity among human haplotypes. (A) Miropeats analysis reveals a great consistency between Haplogroup 5 and the gapped 7q35 TCAF locus in the human reference genome (GRCh38). Colored arrows are annotated TCAF SDs and lines connecting the sequences show regions of homology. The dark green rectangle in the sequence-resolved, gap-free Haplogroup 5 contig (bottom) represents the region missing in the GRCh38 sequence (dark green bar, top). Additional annotations include a schematic of TCAF sequence structure and gene models/isoforms for nonhuman primate, RefSeq gene track (GRCh38), segmental duplication (SegDup) tracks, BAC clones used in the Haplogroup 5 assembly, and predicted TCAF models and isoforms using full-length non-chimeric transcripts from six human tissues (Methods). Note that the TCAF2A gene model in GRCh38 is incorrect due to the presence of the gap in the middle of the TCAF SD region. Also note that the numbers above overlap between two BAC clones and indicate the percent sequence identity (\#identical bases/total bases). For illustration, all predicted gene models and isoforms are aligned to Haplogroup 5 sequence for (B) $T C A F 2 A$ and (C) $T C A F 2 C$, along with annotations for amino acid sequence lengths and haplogroups in which they are observed. For simplicity, we skip sequences between TCAF2A and TCAF2C2 (dashed line). Detailed haplotype-specific and/or tissue-specific gene models and isoforms can be found in Figures S6-S13 and Table S5. 
A

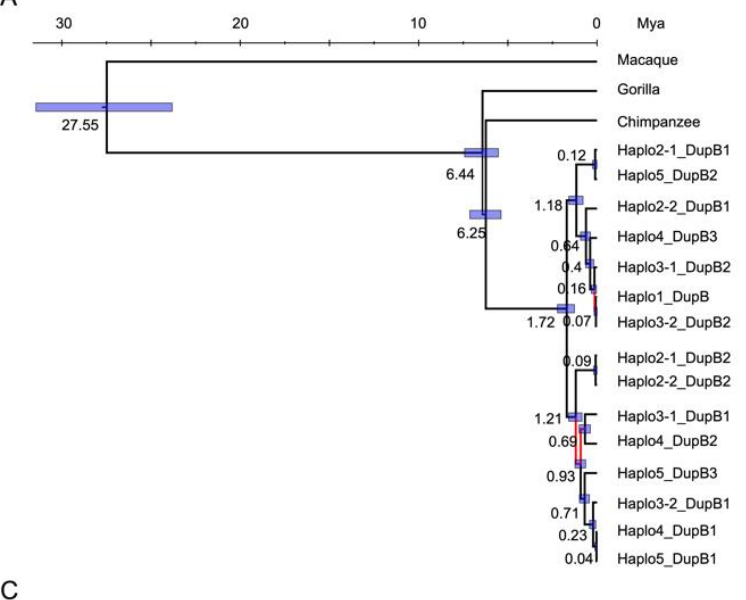

B

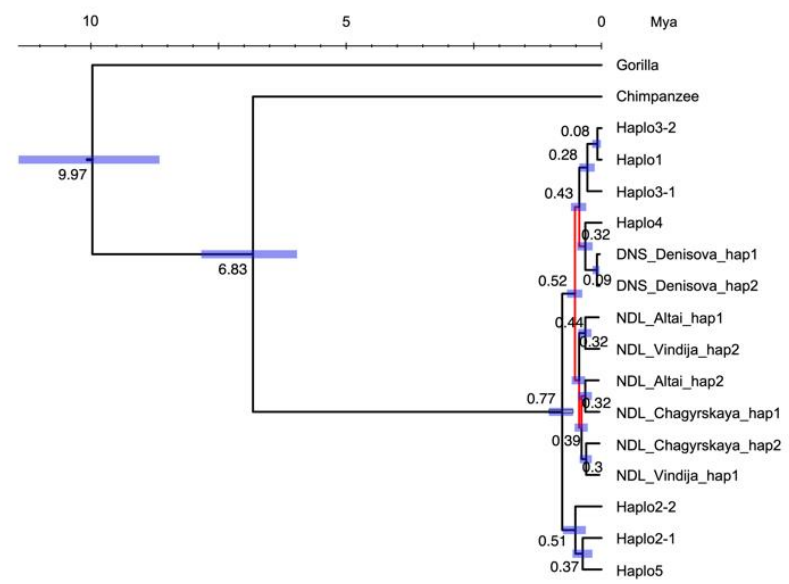

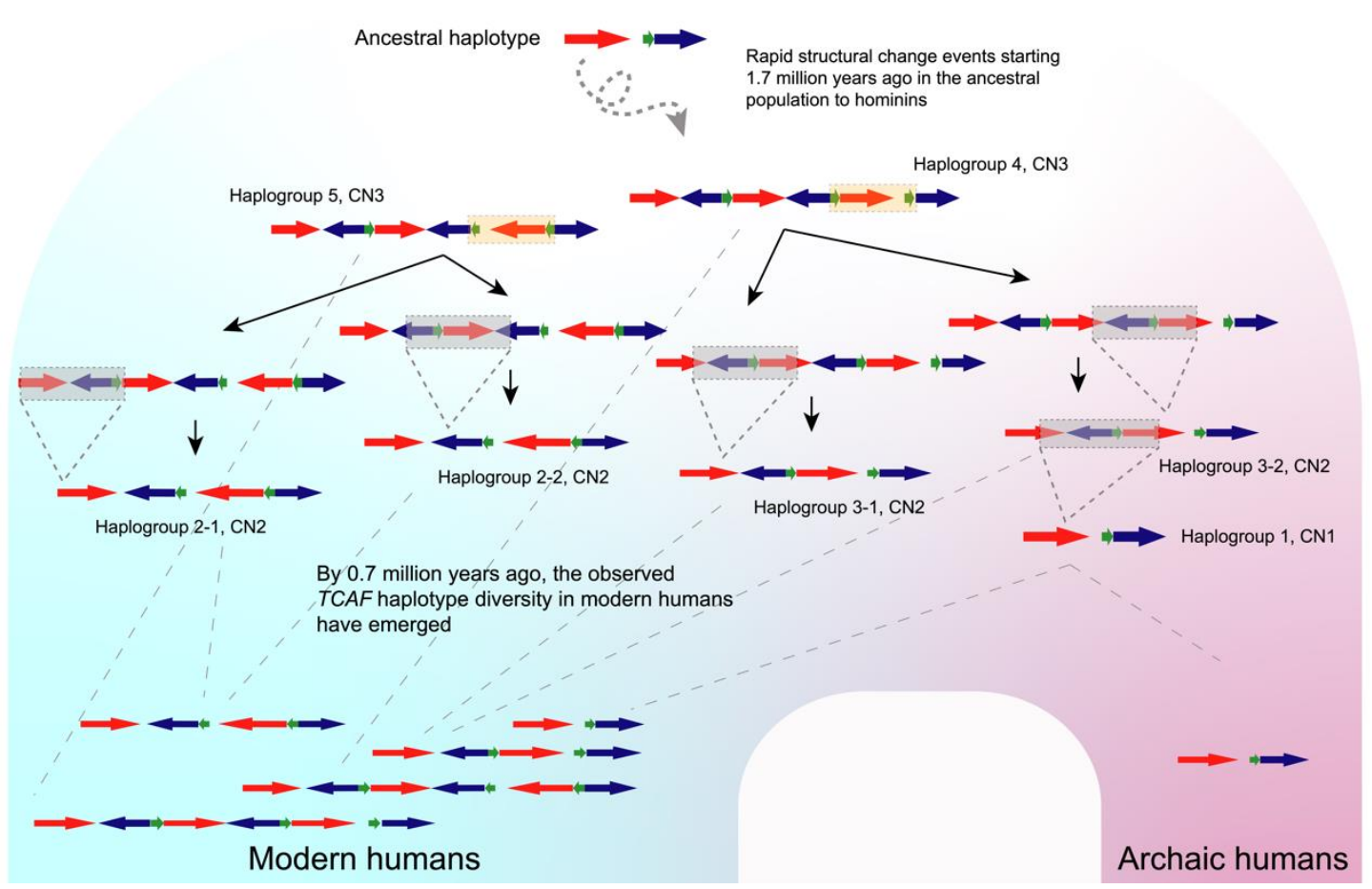

Figure 4. Evolutionary reconstruction of TCAF structural diversity. (A) Phylogeny of the haplogroups was inferred using TCAF DupB sequences and BEAST (v.2.6.2) with five independent runs of 10 million iterations of Markov Chain Monte Carlo (Methods). Numbers and horizontal bars at internal nodes indicate point estimates and $95 \%$ highest posterior density intervals for the divergences (in million years ago, Mya), respectively. Branches with posterior probabilities $<90 \%$ are colored in red. See Figure S14-S16 for results of other SD sequences. (B) Inferred phylogeny of the modern human haplogroups and archaic hominin haplotypes using the $12 \mathrm{kbp}$ unique sequences embedded within TCAF SDs (Figure 1). Haplotypes of archaic samples were generated using high-confident single-nucleotide variants (SNVs) called within the unique diploid region. Phylogenetic inference was performed similarly as described above. (C) Schematic model for the evolution of TCAF haplotypes in humans based on phylogenetic inferences (Figures 4A-4B and S14-S17). Colored arrows are TCAF SDs; orange and gray areas indicate relative inversion and deletion events between haplogroups, respectively. Short dashed lines indicate putative breakpoints of structural changes between haplogroups, while the long-dashed lines illustrate lineage sorting. 
A

- African $\square$ EastAsian $\square$ Melanesian $\square$ Siberian $\square$ NDL

- American $\square$ European $\square$ MiddleEastern $\square$ SouthAsian $\square$ DNS
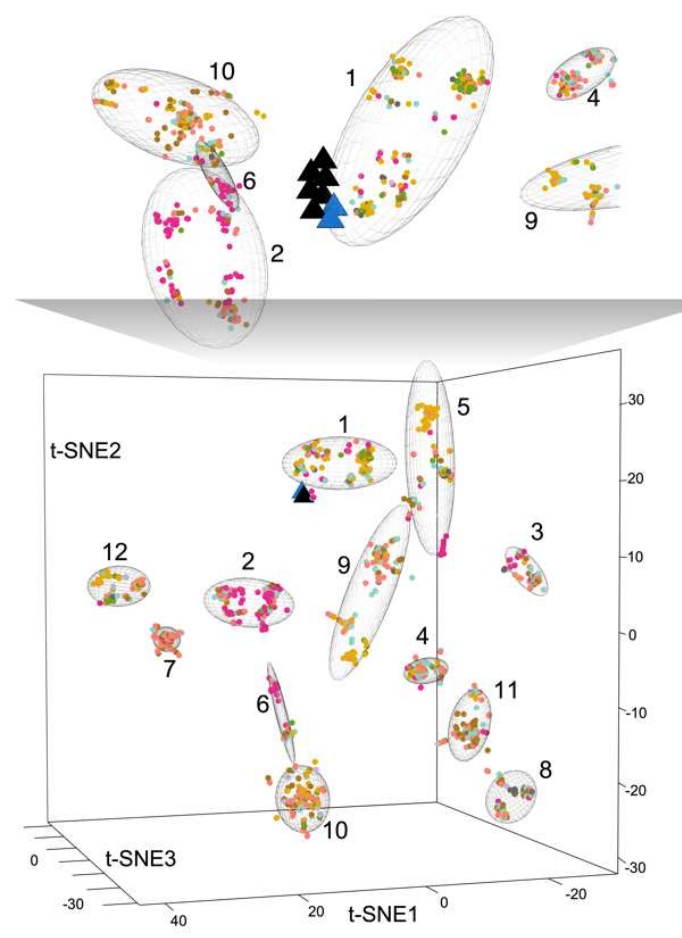

B

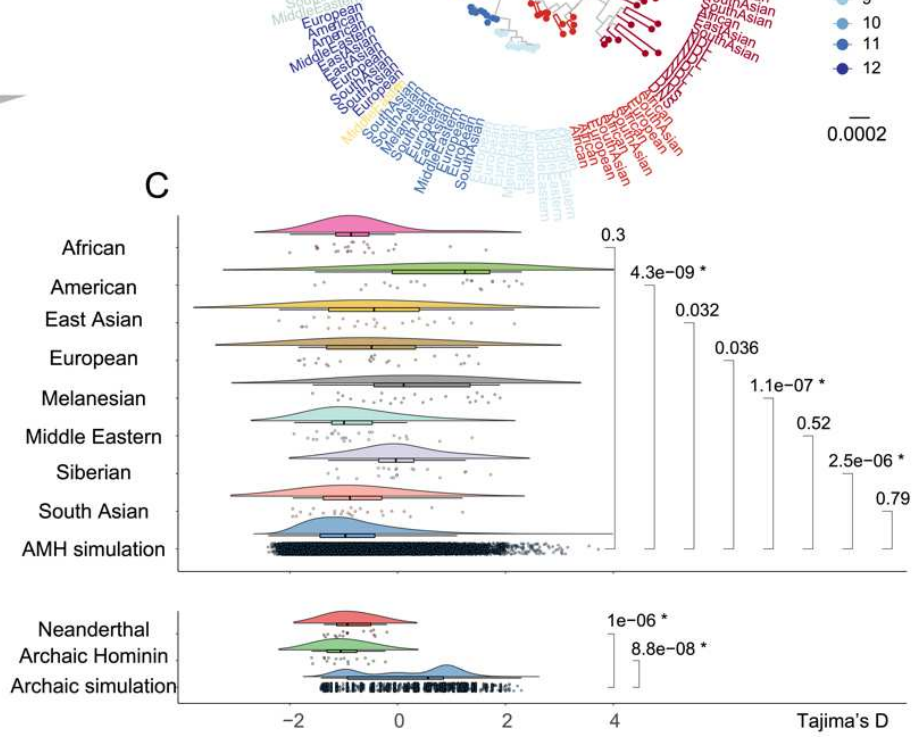

Figure 5. Archaic hominin versus human haplotype diversity. Haplotypes were inferred using 1,275 $\mathrm{SNVs}$ in the three unique diploid sequences around the TCAF SD region (Figures 1 and 2).

(A) Haplotype-based principle component analysis was performed, followed by haplotype clustering and cluster visualization using the dimension-reduction technique, t-SNE (Methods). On the t-SNE plot, each dot/triangle is a haplotype and colored according to population/species origin. NDL and DNS refer to Neanderthal (black triangle) and Denisovan (blue triangle) haplotypes. Numbers and ellipses in the 3D tSNE plots indicate individual clusters (see also Figures S24-S29). The zoom-in above the 3D t-SNE shows that all archaic haplotypes are in tight proximity to each other. (B) The maximum likelihood phylogeny was constructed using 10 randomly selected haplotypes from the 12 inferred clusters, in addition to eight archaic and one chimpanzee haplotypes. Note that the branch length of chimpanzee (dashed line) is truncated by $90 \%$ of its actual length for the purpose of illustration. (C) Distributions of Tajima's D statistic computed using $2 \mathrm{kbp}$ windows sliding over the three unique diploid regions. Significance of natural selection signals was determined using coalescent simulations based on 1,000 different demographic models (Methods). The asterisks indicate significant tests after the Bonferroni correction. 

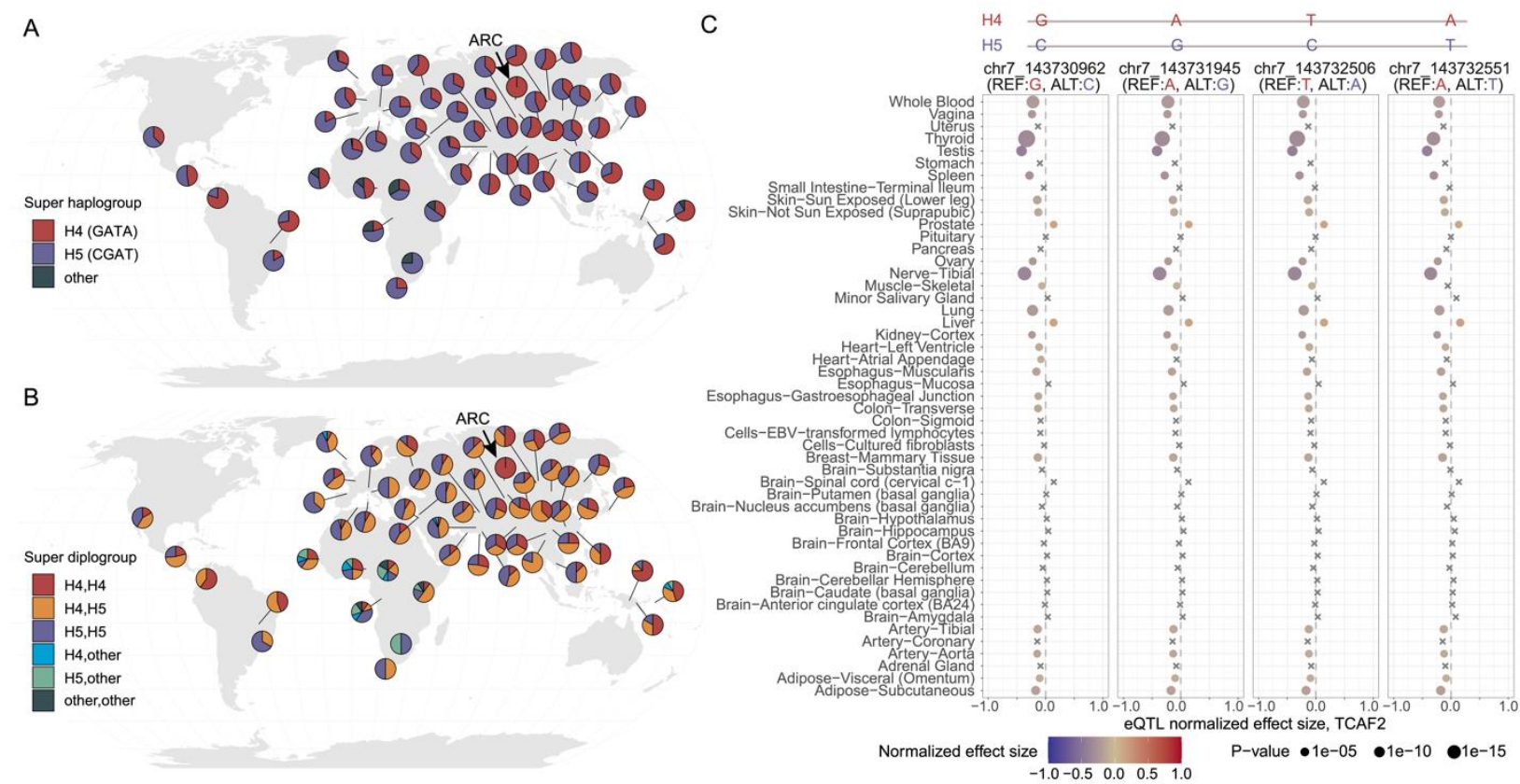

Figure 6. H4 and H5 super haplogroup distribution and eQTL analysis. Four tagging SNVs were first identified by perfectly separating super haplogroups $\mathrm{H} 4$ and $\mathrm{H} 5$ among the seven BAC haplogroups and confirmed based on patterns of linkage disequilibrium (LD) in samples from the HGDP panel. (A and B) Distributions of super haplogroups H4 (red, 57.1\%) and H5 (blue, 40.3\%) and their diploid type in the HGDP populations across the world. Other haplotypes were found in $\sim 2.6 \%$ of the samples. Note that archaic hominin haplotypes from the Neanderthal $(n=3)$ and Denisovan $(n=1)$ samples all carry H4 haplotypes, and as a representation the geographic location of these samples were placed in the Altai Mountains in Siberia (arrows). (C) Multi-tissue eQTL plots show consistent patterns of associations between the four tagging SNVs and expression levels of TCAF2 across 50 tissues. Effect sizes were calculated as the effect of the alternative allele (blue) relative to the reference allele (read) as defined in GTEx (release v8) and are scaled using color. The (unadjusted) p values of eQTL association are represented by dot sizes. Note that crosses (x) indicate insignificant associations. 


\section{Figures}

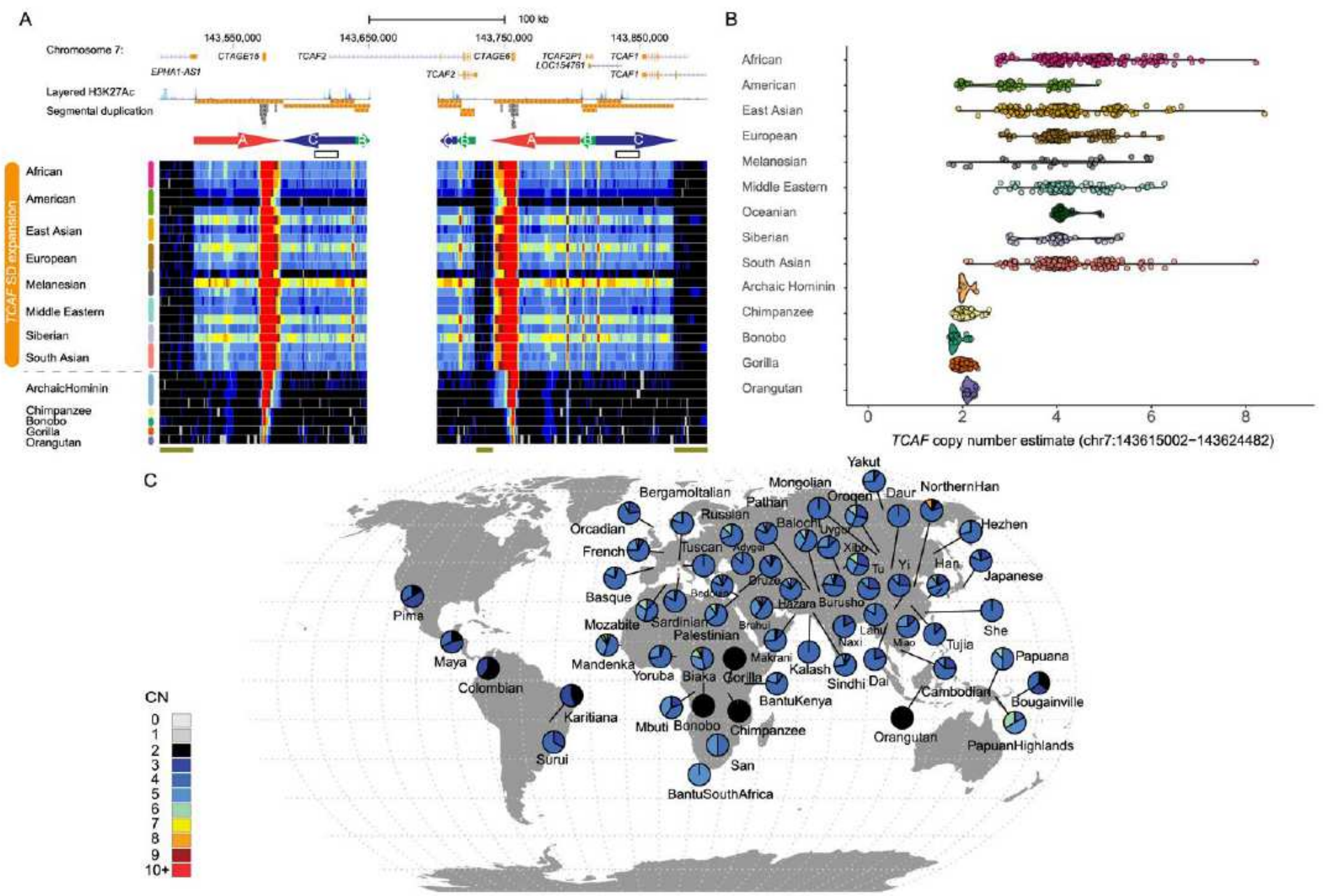

\section{Figure 1}

Copy number variation of TCAF SDs in a collection of diverse human and nonhuman samples. Copy numbers were estimated using read-depth based genotyping method. (A) Copy number (CN) heat maps, where each row represents the $C N$ of a sample over the TCAF locus. The colored arrows $(A, B$, and $C)$ represent the three major SD blocks in this region. The white area in the middle represents the gap present in the human reference genome (GRCh38). The two white boxes show a putative interlocus gene conversion event that correlates with latitudinal locations of populations (Figure S16-S18). Dark green bars at the bottom indicate the unique diploid sequences used for downstream phylogenetic and population genetic analyses (chr7:143,501,000-143,521,000, chr7:143,729,525-143,741,525, chr7:143,875,000-143,895,000 [GRCh38]). (B) Distributions of the overall TCAF CN genotypes among samples from nonhuman great apes, archaic hominins, and modern humans using a representative region (chr7:143,615,002-143,624,482). (C) Geographic distribution of the overall TCAF CN genotypes in the 54 Human Genome Diversity Project (HGDP) populations and the nonhuman great ape samples. Geocoordinates for the populations are listed in Table S8. Pie charts shows the CN frequency distribution for a given population (colors correspond to those in the $\mathrm{CN}$ heat maps). Note: The designations employed 
and the presentation of the material on this map do not imply the expression of any opinion whatsoever on the part of Research Square concerning the legal status of any country, territory, city or area or of its authorities, or concerning the delimitation of its frontiers or boundaries. This map has been provided by the authors.
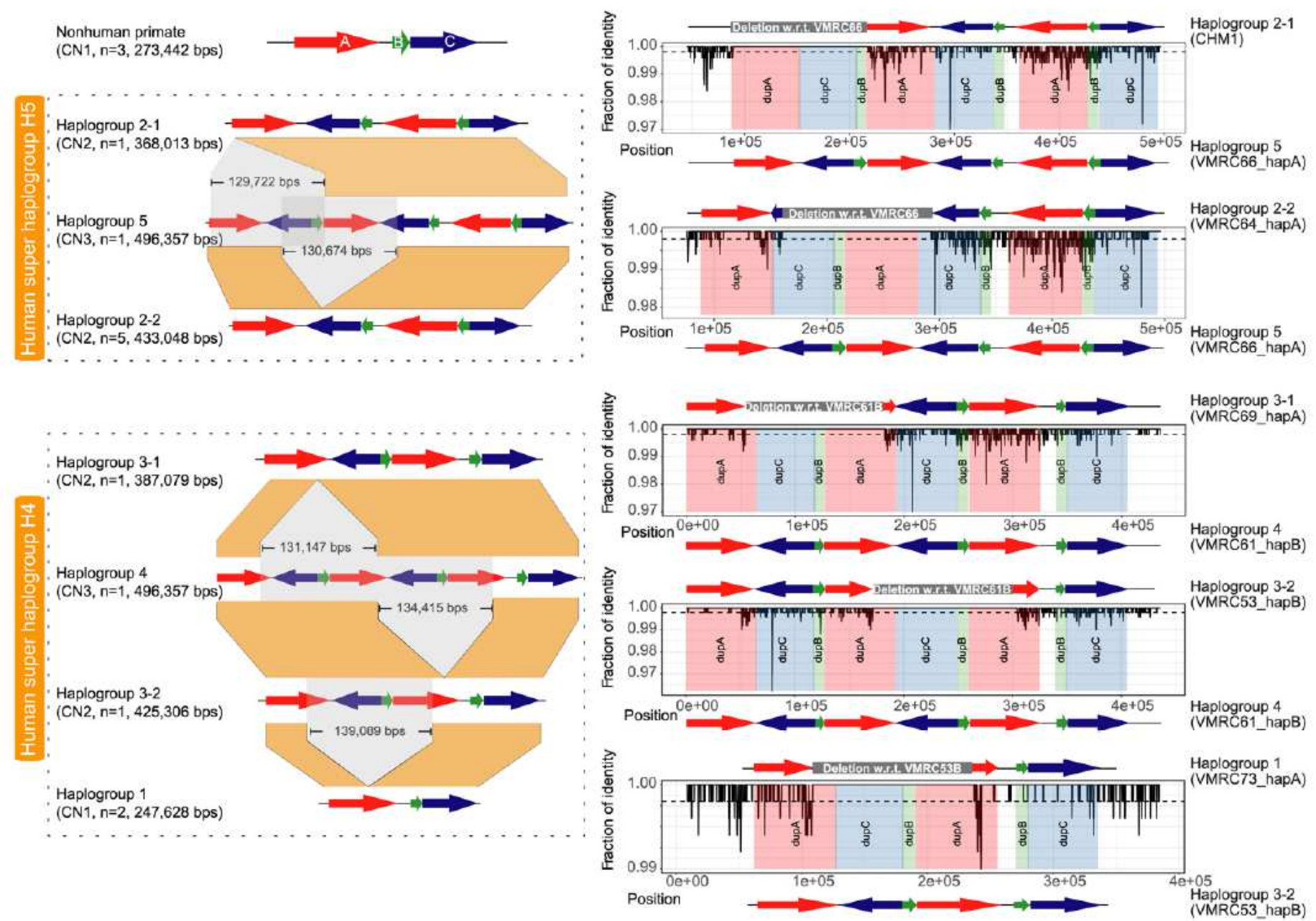

\section{Figure 2}

Complex structural haplotype diversity at the 7q35 TCAF locus in humans. Left panel: Schematic representations of the major- (super haplogroups $\mathrm{H} 4$ and $\mathrm{H} 5$ ) and sub-haplogroups identified from 15 targeted BAC long-read assemblies. Colored arrows are TCAF SDs, and the gray and orange areas indicate putative deletion events and orthologous sequences between haplogroups. For example, despite the similarity in structure, Haplogroup 2-1 and Haplogroup 2-2 are likely resulted from different deletion events from Haplogroup 5 based on comparative sequence data. Right panel: The comparative sequence analysis between individual haplogroups shows pairwise sequence identities (black lines) over 500 bp windows (sliding by 100 bp). With each plot, colored rectangles correspond to TCAF SDs, while the white region embedded between a DupA (red) and a DupB (green) blocks represents a $12.3 \mathrm{kbp}$ single-copy unique sequence. Gray rectangles above each sequence identity plots indicate the locations of putative structure variants (Table S3). 
A

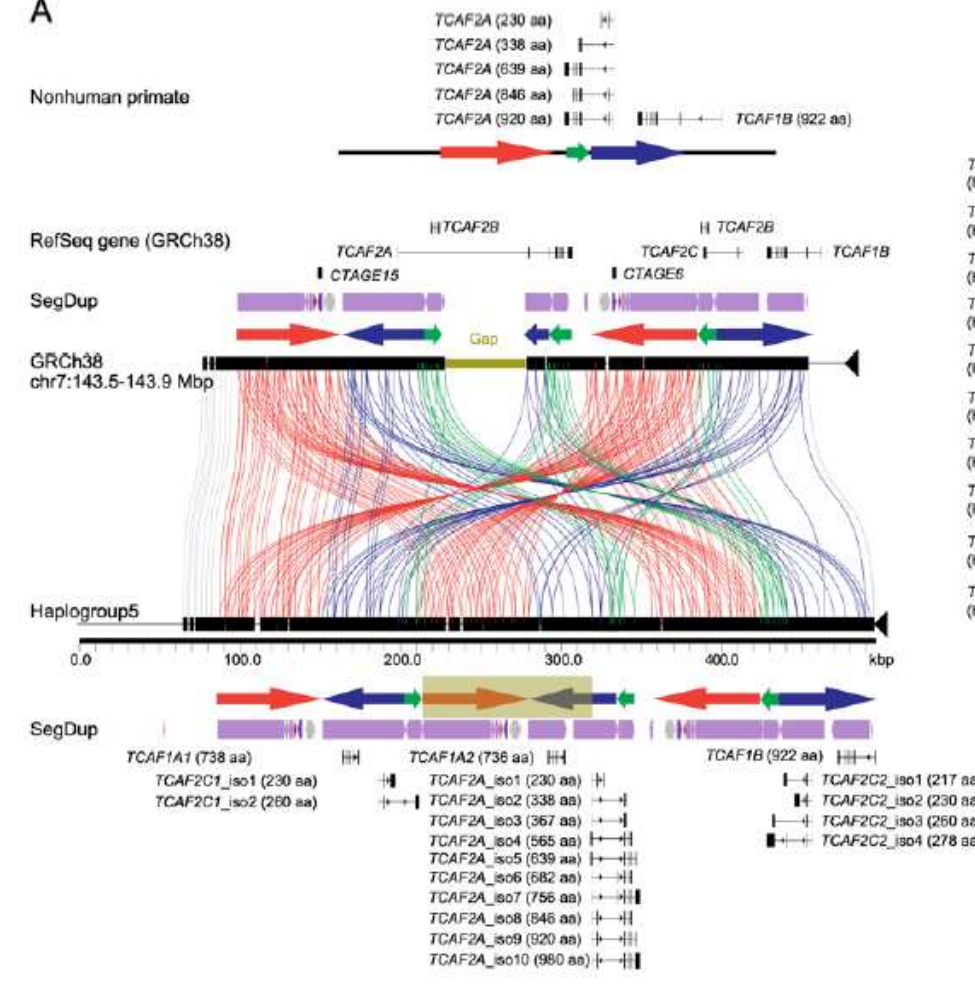

B

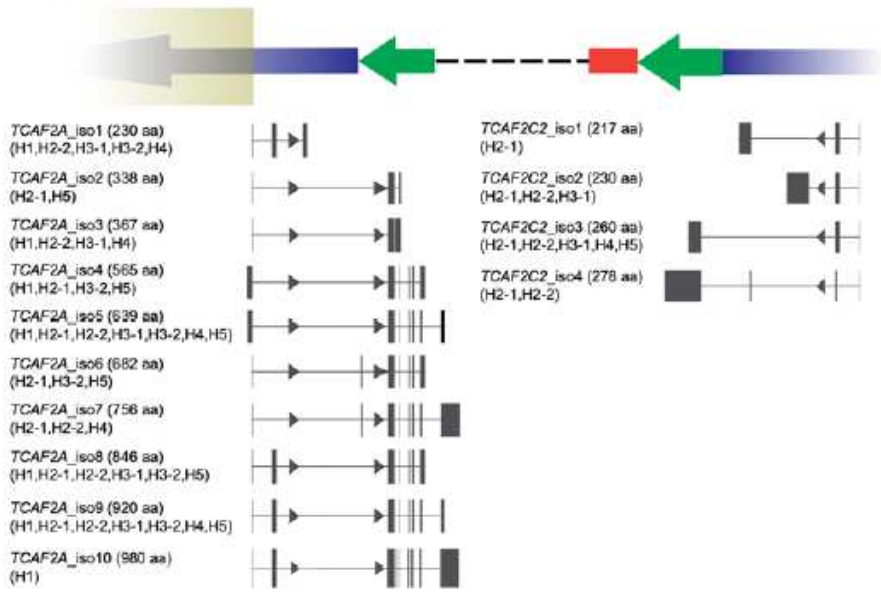

C

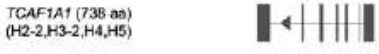

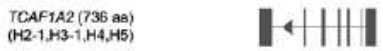

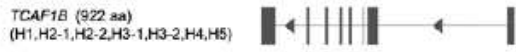

Figure 3

TCAF gene and isoform diversity among human haplotypes. (A) Miropeats analysis reveals a great consistency between Haplogroup 5 and the gapped 7q35 TCAF locus in the human reference genome (GRCh38). Colored arrows are annotated TCAF SDs and lines connecting the sequences show regions of homology. The dark green rectangle in the sequence-resolved, gap-free Haplogroup 5 contig (bottom) represents the region missing in the GRCh38 sequence (dark green bar, top). Additional annotations include a schematic of TCAF sequence structure and gene models/isoforms for nonhuman primate, RefSeq gene track (GRCh38), segmental duplication (SegDup) tracks, BAC clones used in the Haplogroup 5 assembly, and predicted TCAF models and isoforms using full-length non-chimeric transcripts from six human tissues (Methods). Note that the TCAF2A gene model in GRCh38 is incorrect due to the presence of the gap in the middle of the TCAF SD region. Also note that the numbers above overlap between two BAC clones and indicate the percent sequence identity (\#identical bases/total bases). For illustration, all predicted gene models and isoforms are aligned to Haplogroup 5 sequence for (B) TCAF2A and (C) TCAF2C, along with annotations for amino acid sequence lengths and haplogroups in which they are observed. For simplicity, we skip sequences between TCAF2A and TCAF2C2 (dashed line). Detailed haplotype-specific and/or tissue-specific gene models and isoforms can be found in Figures S6-S13 and Table S5. 
A

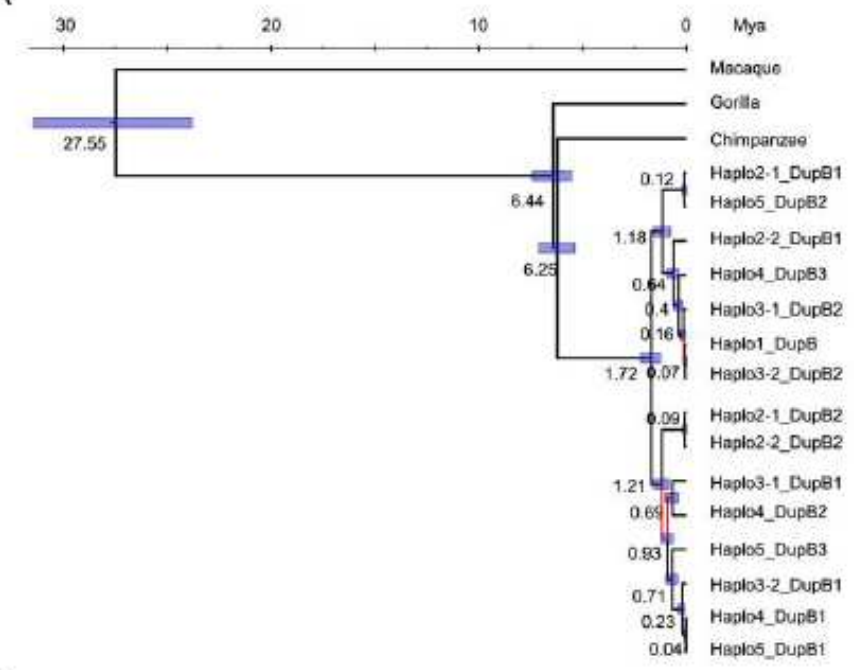

B

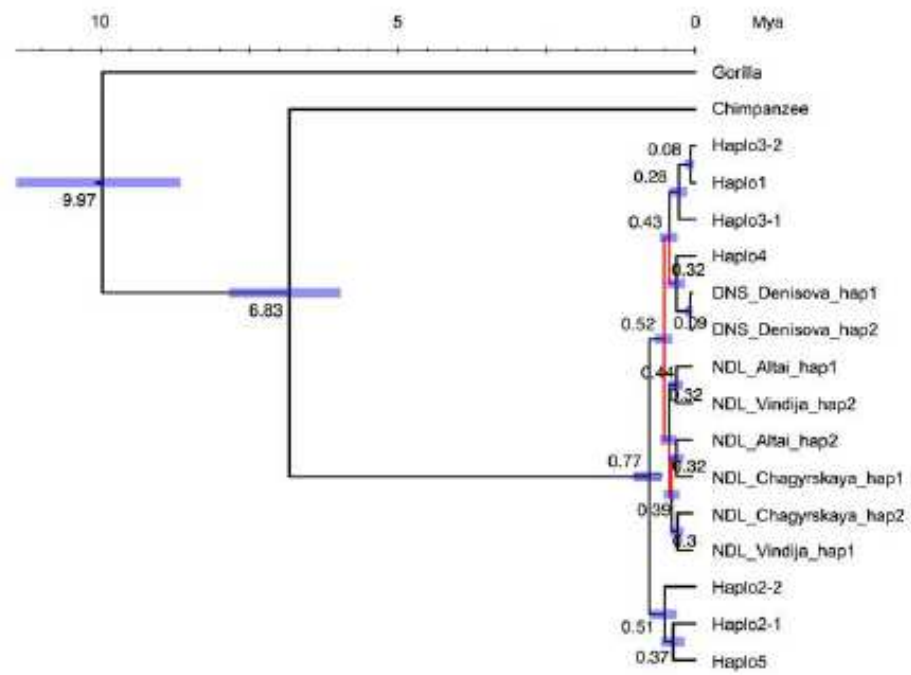

C

Ancestral haplotype

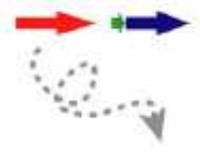

Rapid structural change events starting

1.7 million years ago in the ancestral

population to hominins

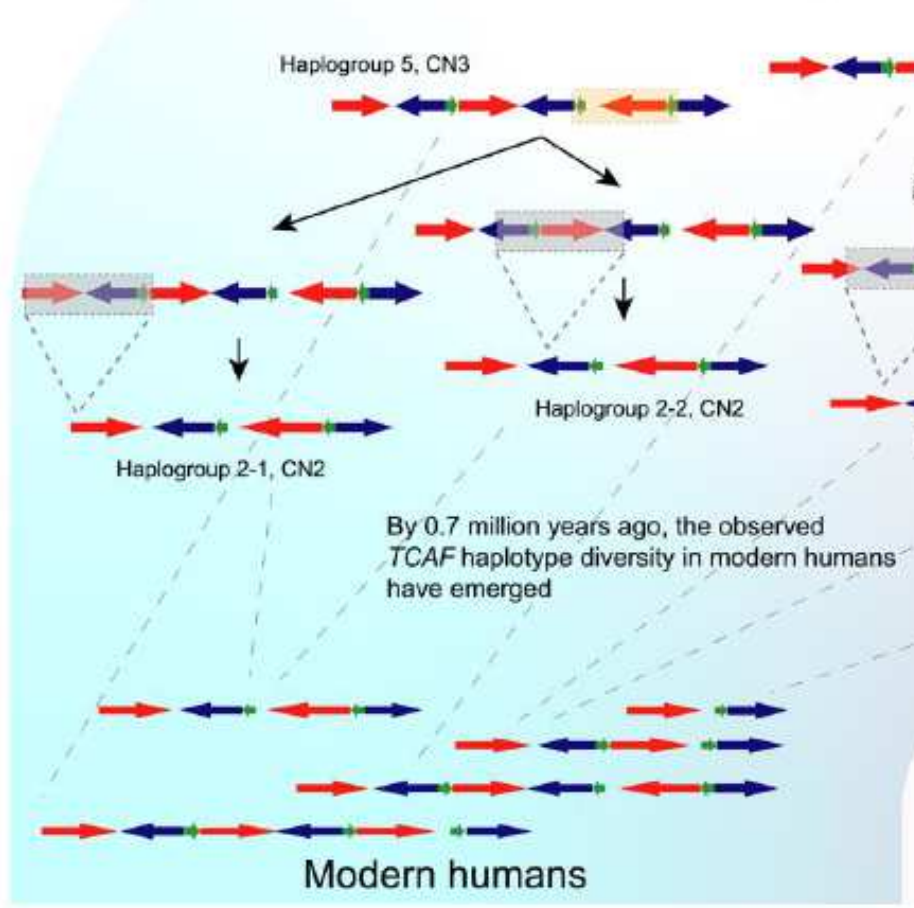

Hapiogroup 4, CN3
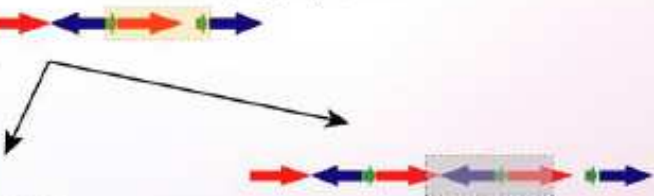

$\downarrow$

Haplogroup 3-1, CN2

Haplogroup 1, CN1

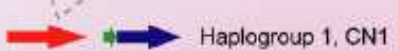

By 0.7 million years ago, the observed

TCAF haplotype diversity in modern humans

have emerged
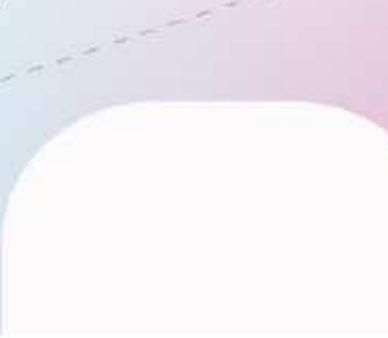

Archaic humans

\section{Figure 4}

Evolutionary reconstruction of TCAF structural diversity. (A) Phylogeny of the haplogroups was inferred using TCAF DupB sequences and BEAST (v.2.6.2) with five independent runs of 10 million iterations of Markov Chain Monte Carlo (Methods). Numbers and horizontal bars at internal nodes indicate point estimates and $95 \%$ highest posterior density intervals for the divergences (in million years ago, Mya), respectively. Branches with posterior probabilities $<90 \%$ are colored in red. See Figure S14-S16 for results of other SD sequences. (B) Inferred phylogeny of the modern human haplogroups and archaic hominin haplotypes using the $12 \mathrm{kbp}$ unique sequences embedded within TCAF SDs (Figure 1). Haplotypes of archaic samples were generated using high-confident single-nucleotide variants (SNVs) called within the 
unique diploid region. Phylogenetic inference was performed similarly as described above. (C) Schematic model for the evolution of TCAF haplotypes in humans based on phylogenetic inferences (Figures 4A-4B and S14-S17). Colored arrows are TCAF SDs; orange and gray areas indicate relative inversion and deletion events between haplogroups, respectively. Short dashed lines indicate putative breakpoints of structural changes between haplogroups, while the long-dashed lines illustrate lineage sorting.

A

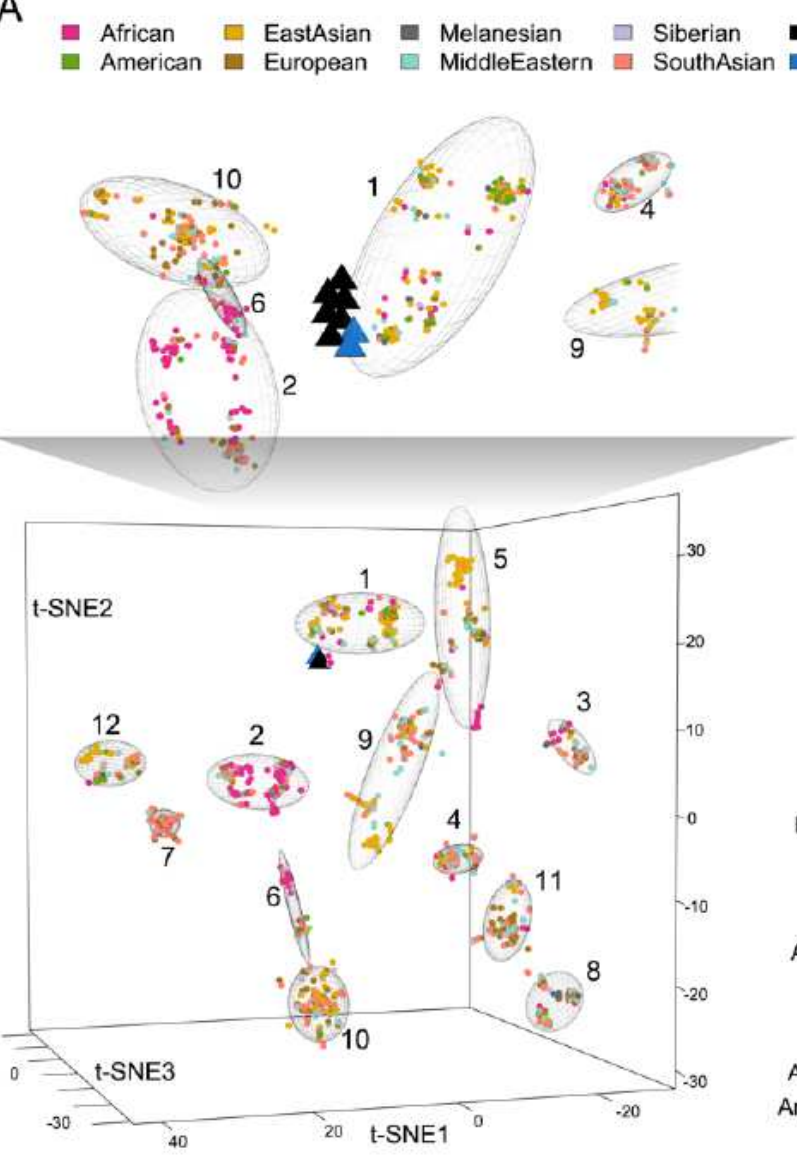

B

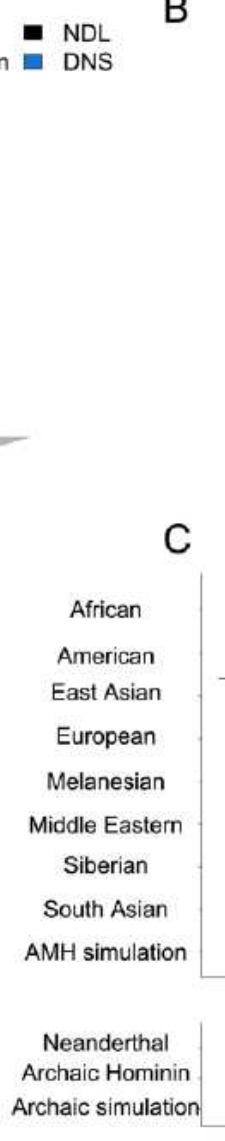

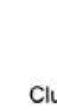

Cluster

- 0

- 1

2
$-\quad 3$

- 4

- 5

$\begin{array}{r}6 \\ -7 \\ \hline\end{array}$

8

- 9
- 10

- 11

- 12

$0 . \overline{0002}$
C

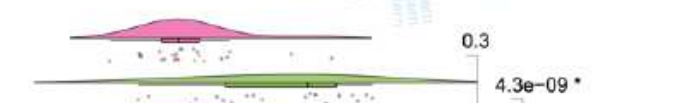

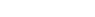


determined using coalescent simulations based on 1,000 different demographic models (Methods). The asterisks indicate significant tests after the Bonferroni correction.

A

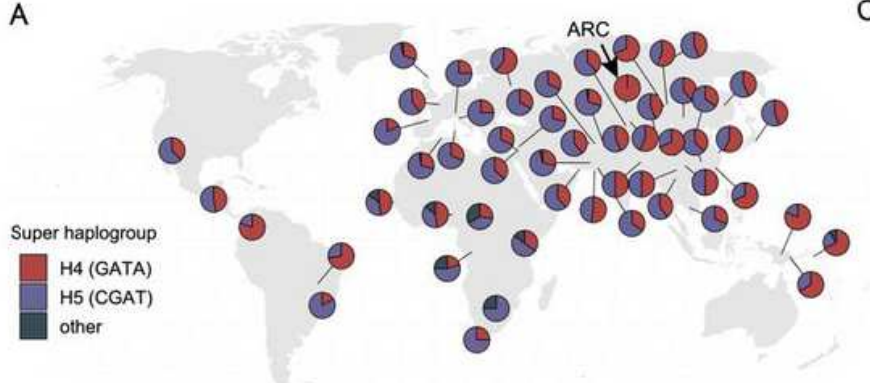

B

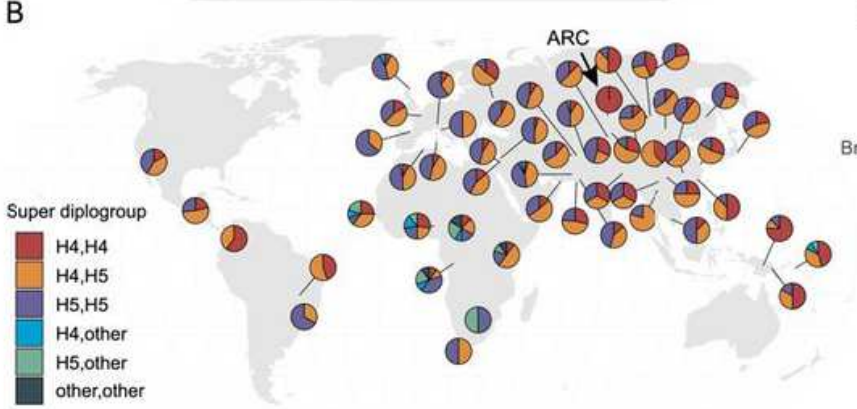

C
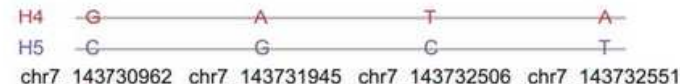
chr7 143730962 chr7 143731945 chr7 143732506 chr7 143732551

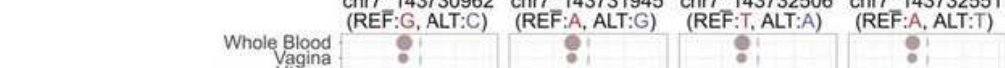
Thyroid
Testis
Stomach
Spleen
Small Intestine-Terminal leum
Skin-Sun Exposed LLower leg
Skin-Not Sun Exposed (Suprapubic)
Prostete
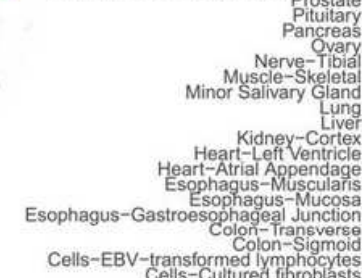

Cells-EBV-transformed lymphongcytes
Cells-Cultured fibroblasts
Breast-Mammary Tissue Brain-Spinal cord (cervical c-1) Brain-Spinal cord (cervical c-1)
Brain-Putamen (basal ganglia Bain-Nucleus accumbens
Brain-Hypal ganghilia

Brain-Hippocampus
Brain-Frontal Cortex (BA9) Brain-Cerebellum
Brain-Cerebellar Hermisphere Brain-Cerebellar Hemisphere
Brain-Caudate (basal ganglia)

Brain-Caudate (basal ganglia)
Anterior cingulate cortex (BA24)
Brain-Amygdala

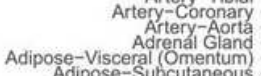

Adrenal Gland
Adipose-Visceral (Omentum)
Adipose-Subcutaneous

effect size

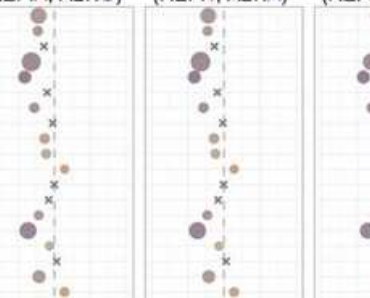

?

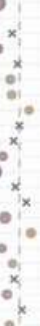

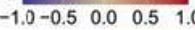

$a^{x}$

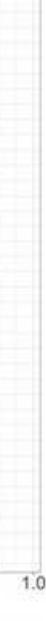

\section{Figure 6}

$\mathrm{H} 4$ and $\mathrm{H} 5$ super haplogroup distribution and eQTL analysis. Four tagging SNVs were first identified by perfectly separating super haplogroups $\mathrm{H} 4$ and $\mathrm{H} 5$ among the seven BAC haplogroups and confirmed based on patterns of linkage disequilibrium (LD) in samples from the HGDP panel. (A and B) Distributions of super haplogroups H4 (red, $57.1 \%$ ) and H5 (blue, $40.3 \%$ ) and their diploid type in the HGDP populations across the world. Other haplotypes were found in $\sim 2.6 \%$ of the samples. Note that archaic hominin haplotypes from the Neanderthal $(n=3)$ and Denisovan $(n=1)$ samples all carry H4 haplotypes, and as a representation the geographic location of these samples were placed in the Altai Mountains in Siberia (arrows). (C) Multi-tissue eQTL plots show consistent patterns of associations between the four tagging SNVs and expression levels of TCAF2 across 50 tissues. Effect sizes were calculated as the effect of the alternative allele (blue) relative to the reference allele (read) as defined in GTEx (release v8) and are scaled using color. The (unadjusted) p values of eQTL association are represented by dot sizes. Note that crosses $(x)$ indicate insignificant associations. Note: The designations employed and the presentation of the material on this map do not imply the expression of any opinion whatsoever on the part of Research Square concerning the legal status of any country, territory, city or area or of its authorities, or concerning the delimitation of its frontiers or boundaries. This map has been provided by the authors.

\section{Supplementary Files}


This is a list of supplementary files associated with this preprint. Click to download.

- TCAFsupplementaryFiguresandTables.v8.pdf

- TableS1VSTpairwiseSuperPop.sorted.v8.xIsx

- TableS7GENECONVIGCtracks.v8.xlsx

- TableS8GeographicCoordinatesHGDPpopsandCNsummary.v8.xlsx 